\title{
Assessing the ecological status of fluvial ecosystems employing a macroinvertebrate multi-taxon and multi-biomarker approach
}

\author{
Carolina Rodrigues,
}

Ana Bio,

Laura Guimarães,

Virgínia C. Fernandes,

Cristina Delerue-Matos \&

Natividade Vieira

Abstract

Biomarkers are recognised sensitive early-warning tools of biological effects in aquatic organisms. In this scope, the main aim of this study was to investigate the potential usefulness of a battery of biomarkers, evaluated in different benthic macroinvertebrate taxa, to discriminate aquatic ecosystems with different levels of ecological status and to provide further clues supporting environmental management. The study took place during the autumn of 2013 and the spring and summer of 2014, and the study cases were two Mediterranean rivers (Âncora and Ferreira rivers), differing in their ecological status. The biomarkers determined are widely employed and comprise a large set of biochemical responses: the activity of enzymes (cholinesterases, glutathione $S$-transferases, catalase and lactate dehydrogenase) and the levels of lipid peroxidation. They were assessed seasonally and in different macroinvertebrate taxa. Thirteen water physico-chemical parameters were also seasonally determined, and the concentration of seven organophosphorus pesticides and the percentage of 32 trace metals in sediments were determined in the spring. This is particularly useful for water management. Based on this, authorities can take actions to prevent further damage in the ecological status. Multivariate analyses showed distinct patterns of biological response for the Calopteryx spp., Chironomidae and Baetis spp. taxa. Calopteryx spp. and Chironomidae, in particular, showed distinct response patterns for the two rivers, which were fairly stable across seasons. This study sets the foundations for future cost-effective biomonitoring campaigns in Mediterranean rivers, allowing to establish historical data important to understand ecosystem evolution, as well as baseline levels of diagnostic biomarkers in informative macroinvertebrate taxa.

\section{Introduction}

The EU Water Framework Directive (WFD; EC 2000) is the main legal instrument for the management, protection and restoration of aquatic ecosystems. It changed conceptually the water management in EU Member States, through the use of ecosystem health and sustainability principles as a basis for decision-making. Nowadays, biological 
quality elements (BQEs), integrating the effects of all stressors, are used to evaluate the ecological status of surface waters. Because ecosystems are so diverse, and complex, fluctuating entities, one of the most technically challenging aspects faced in the application of the WFD is the development of systems for adequate ecological assessment and classification.

For the assessment of fluvial ecosystems, indices based on the analysis of fish, macrophytes, phytobenthos and benthic macroinvertebrates are the BQEs recommended by the WFD. Each of these indices relies on surveys of the community structure, for the respective BQEs, to evaluate and classify a rivers' ecological quality. They detect relevant changes that usually cause the elimination of more sensitive species from a particular site (Damásio et al. 2011). While having high ecological relevance, because of the loss of biodiversity, these indices are of limited interest to anticipate specific protection measures required to maintain ecological quality or prevent its damage. The necessity for tools that are fast and sensitive enough to show sub-lethal alterations in aquatic organisms, able to anticipate future detrimental ecological effects, has raised interest in biomarkers, as useful tools to complement the information from indices based on the analysis of the community structure. Measured at individual or sub-individual levels of biological organisation, biomarker responses occur on shorter timescales. In particular, multi-biomarker evaluations can reveal early signs of exposure and possible adverse outcomes, translating the integrated impact of chemical contaminants and natural stressors which animals are undergoing (Allan et al. 2006; Hagger et al. 2006). The incorporation of macroinvertebrate biomarkers in the biomonitoring of rivers and streams has thus been increasingly reported (e.g. Olsen et al. 2001; Berra et al. 2004; Bonzini et al. 2008; Minutoli et al. 2013; De Castro-Catala et al. 2015). However, studies aiming to evaluate the ecological quality of rivers using both biomarker- and community-based approaches in macroinvertebrates are still rare (Barata et al. 2005; Puertolas et al. 2010; Damásio et al. 2011; Prat et al. 2013). Most of these studies were based on the determination of biomarkers from a single and tolerant macroinvertebrate species. Some authors, however, have found that, depending on the species selected, the evaluation using a sole species may lead to misleading over- or under-estimation of the risk (Berra et al. 2004; Bonzini et al. 2008). These authors determined biochemical biomarkers in different macroinvertebrate taxonomic groups, mainly in families of the Amphipoda, Diptera, Ephemeroptera, Isopoda, Odonata, Plecoptera and Trichoptera orders (Berra et al. 2004; Bonzini et al. 2008), rather than in one single species. Their studies report differential taxa sensitivities for several biomarkers (e.g. involved in neurotransmission, biotransformation and antioxidant defences) (Berra et al. 2004; Bonzini et al. 2008), suggesting that particular attention should be paid to the selection of the taxa employed in a given monitoring scheme, so as to avoid biased risk estimations. Though samples obtained for genus and/or families may include species with some differential sensitivity, the above-mentioned results suggest that this approach of using higher levels of taxonomic resolution may be effective in detecting subtle gradients of contamination and their associated effects on the biota. Moreover, from the phylogenetic perspective, species from a given genus (or sister species) probably exhibit likened physiological responses (Colin et al. 2016). Therefore, further studies employing multiple biomarkers in combination with measured environmental stressors, multiple macroinvertebrate taxa and river types are required to evaluate the wider usefulness of this approach. These will also contribute to establish efficient and cost-effective biomarker strategies that can anticipate future ecological damage. In this context, the aims of the present study were (i) to investigate if a battery of biomarkers evaluated in different benthic macroinvertebrate 
taxa could discriminate aquatic ecosystems with different levels of ecological quality; (ii) to understand if biomarker data can help in identifying potential problems or sources of contamination affecting aquatic biota, complementing the information given by ecological quality indices; and (iii) to identify the most favourable taxa and season(s) to be used in a multi-biomarker and multi-taxon analysis in cost-effective biomonitoring programmes. To tackle these objectives, a seasonal study was carried out in two northern Iberian rivers with different ecological status. Thirteen physico-chemical water parameters were measured in both rivers. The concentrations of seven organophosphorus pesticides and the percentages of 32 trace metals in the sediment were also determined in the spring (season of major application of pesticides in agriculture). A battery of biomarkers of neurotoxicity (cholinesterases activity), biotransformation (glutathione $S$ transferases), antioxidant defences (catalase), oxidative damage (lipid peroxidation) and energy metabolism (lactate dehydrogenase) was seasonally assessed in different macroinvertebrate taxa collected at various sites in the two rivers.

\section{Materials and methods}

\section{Study area and sampling sites}

The study was carried out during the autumn of 2013 and the spring and summer of 2014, in two Northern Portuguese rivers integrated in the Natura 2000 Network: Âncora River $\left(41^{\circ} 48^{\prime} 5.63^{\prime \prime} \mathrm{N}, 8^{\circ} 46^{\prime} 28.57^{\prime \prime} \mathrm{W}\right)$ and Ferreira River $\left(41^{\circ} 11^{\prime} 15.06^{\prime \prime} \mathrm{N}, 8^{\circ} 27^{\prime} 25.47^{\prime \prime}\right.$ W) (Fig. 1a). These areas differ in their ecological status, which was determined by Rodrigues et al. (submitted), considering the three biological quality elements (hydromorphological, physico-chemical and biological) recommended by the WFD. According to the authors, in general, Âncora River (AR) sites presented "moderate" ecological status classification in all seasons (except AR5 which achieved the "good" ecological status target in the spring and summer of 2014), while Ferreira River (FR) sites presented ecological status classification from "moderate" to "poor" (Rodrigues et al., submitted). Geographically, the AR (Fig. 타 $)$ springs from Serra de Arga, in Viana do Castelo Municipality, and runs approximately $17.91 \mathrm{~km}$ through a steep bedrock, before flowing directly into the Atlantic Ocean, in Caminha Municipality (PGRH1 2016). The FR (Fig. 1c) springs in Paços de Ferreira Municipality, has an approximate length of 22.30 $\mathrm{km}$ and joins the Sousa River in Gondomar Municipality (PGRH3 2016).

\section{Fig. 1}


a

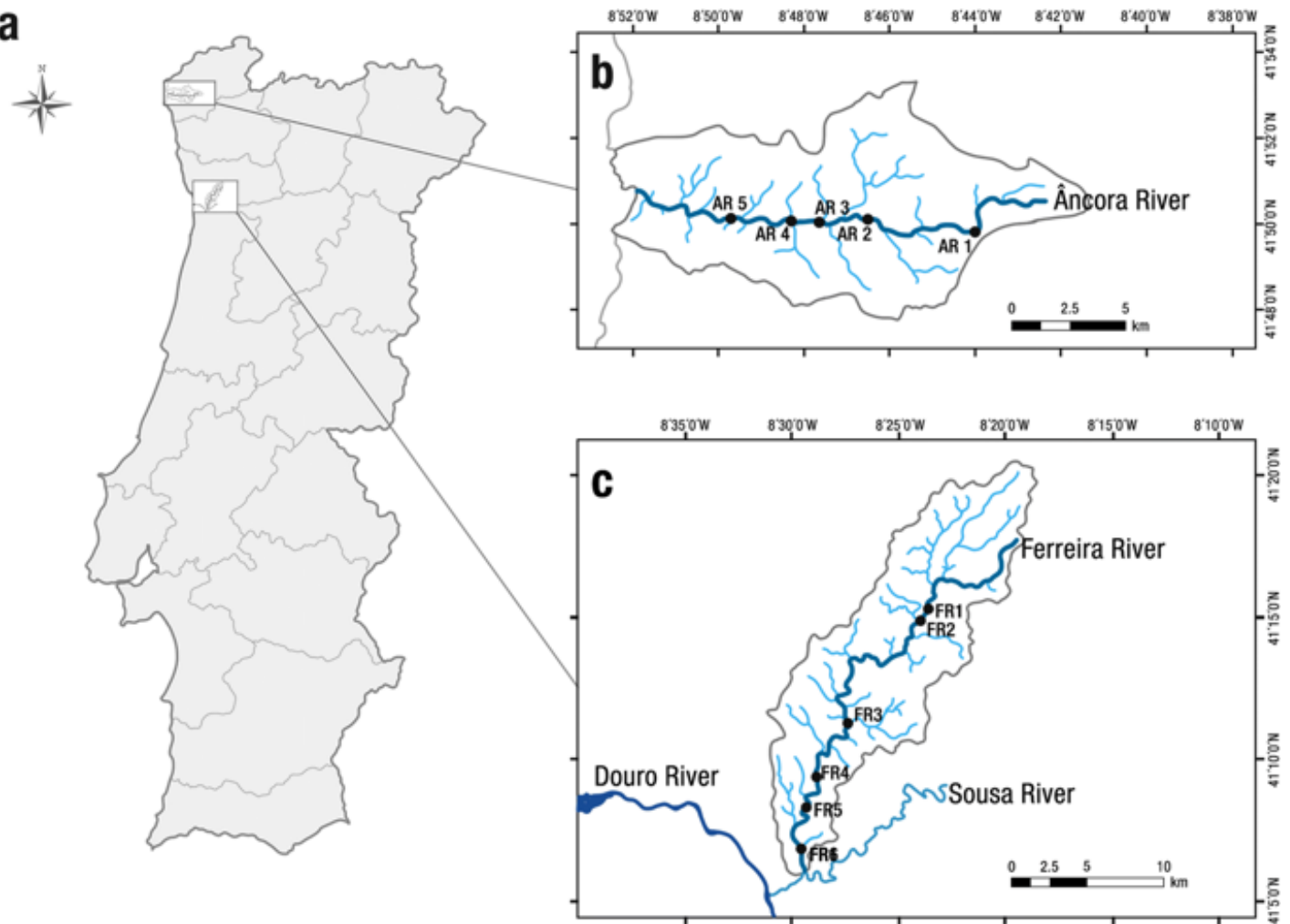

Geographical situation. a Location of the hydrographic basins of the Âncora River and Ferreira River (rectangles), in Portugal mainland. b Map of the hydrographic basin of the Âncora River and the distribution of the sampling sites in the Âncora River (AR1 to AR5). c Map of the hydrographic basin of the Ferreira River and the distribution of the sampling sites in the Ferreira River (FR1 to FR6)

Both the AR and FR belong to the "small-sized streams of the north" national river type (catchment area $<100 \mathrm{~km}^{2}$ ), which reflects the country's northern climate with high annual average precipitation (mean $1190.25 \mathrm{~mm} \pm 357.80 \mathrm{~mm}$ ) and low annual average temperature (mean $12.42{ }^{\circ} \mathrm{C} \pm 1.26^{\circ} \mathrm{C}$ ) (INAG 2008). Both rivers are mainly located in areas with siliceous geology (schist, granite), presenting low mineralisation (INAG 2008). In the hydrographic basin of the AR, the main sector of activity is agriculture (PGRH1 2012), and in the hydrographic basin of the FR, the industrial activity is largely dominant, followed by agriculture.

\section{Physico-chemical, hydromorphological and biological parameters}

Water physico-chemical parameters were seasonally monitored (autumn of 2013 and spring and summer of 2014, i.e. except in winter due to high river discharge during this season), simultaneously with the macroinvertebrate sampling campaigns. The total dissolved solids (TSS, mg/L), $\mathrm{pH}$, water temperature $\left({ }^{\circ} \mathrm{C}\right)$, dissolved oxygen concentration ( $\mathrm{mg} \mathrm{O}_{2} / \mathrm{L}$ ) and percent saturation (\% DO), salinity (PSU) and conductivity $(\mu \mathrm{S} / \mathrm{cm})$ were measured using a multi-parameter portable meter (HI 9829, Hanna Instruments). Nutrient concentrations (nitrates, nitrites, ammonium ion and total phosphorus in $\mathrm{mg} / \mathrm{L})$, the chemical oxygen demand (COD) value $(\mathrm{mg} / \mathrm{L})$ and the total suspended solids $(\mathrm{mg} / \mathrm{L})$ were determined in the laboratory. COD and nutrients were 
determined using a Hanna Instruments multi-parameter bench photometer following the manufacturer's protocols (Hanna Instruments 2014), and TSS was measured through the method described by the American Public Health Association (APHA 1992). Physicochemical parameters were classified, considering the maximum limit values established for the "good" ecological status in Northern Portuguese rivers (INAG 2009).

The scores of the hydromorphological and biological indices, determined for the same sites and seasons for which organisms for biomarker analyses were collected (obtained from Rodrigues et al., submitted), were used for statistical tests and multivariate analysis. The biological quality of sampling sites was assessed through the North Invertebrate Portuguese Index, which evaluates the local community of benthic macroinvertebrates $\left(\right.$ IPtI $_{\mathrm{N}}$ ) (INAG 2009; EC 2013). The hydromorphological quality was assessed through the indices of Habitat Quality Assessment (HQA) and Habitat Modification Score (HMS). For this, the River Habitat Survey (RHS) methodology (Raven et al. 1997, 2002, 2009) was followed. The HQA index provides an indication of the overall habitat diversity in the river channel and riparian corridor, and the HMS index provides a useful indication of possible artificial modifications undergone in the morphology of the river channel.

\section{Analysis of pesticides}

To avoid the influence of rainfall events (e.g. runoff, dilution or infiltration) on pesticide analyses, sediment sampling campaigns were carried out in late spring. Seven organophosphorus pesticides used in agriculture in the studied areas were analysed, namely chlorpyrifos, chlorpyrifos methyl, chlorfenvinphos, diazinon, dimethoate, malathion and parathion methyl. Sediment samples for pesticide analysis were obtained in triplicate from each sampling site; the first centimetre of sediment was collected, as suggested by Rubal et al. (2009), and kept at $-20{ }^{\circ} \mathrm{C}$ until analysis.

\section{Reagents}

Seven organophosphorus pesticide standards (chlorfenvinphos, chlorpyrifos, chlorpyrifos methyl, diazinon, dimethoate, malathion and parathion methyl) and the internal standard (IS) - triphenyl phosphate, with $\geq 99 \%$ purity-were obtained from Sigma-Aldrich (St. Louis, MO, USA). Acetonitrile ( $\mathrm{MeCN})$ and $n$-hexane, with chromatography grade, were from Merck ${ }^{\mathrm{TM}}$ (Darmstadt, Germany).

Quick, Easy, Cheap, Effective, Rugged, and Safe (QuEChERS) tubes contained $1.5 \mathrm{~g}$ sodium citrate dehydrate $\left(\mathrm{Na}_{3} \mathrm{Cit} \cdot 2 \mathrm{H}_{2} \mathrm{O}\right), 1.5 \mathrm{~g}$ sodium chloride $(\mathrm{NaCl}), 6 \mathrm{~g}$ magnesium sulphate $\left(\mathrm{MgSO}_{4}\right)$ and $0.750 \mathrm{~g}$ disodium citrate sesquihydrate $\left(\mathrm{Na}_{2} \mathrm{HCit} \cdot 1.5 \mathrm{H}_{2} \mathrm{O}\right)$ (Correia-Sá et al. 2012). The clean-up tube had $150 \mathrm{mg}$ magnesium sulphate $\left(\mathrm{MgSO}_{4}\right)$, $50 \mathrm{mg}$ PSA and $50 \mathrm{mg} \mathrm{C18}$ (Correia-Sá et al. 2012). The clean-up tubes and the QuEChERS were purchased from UCT® (Bristol, PA, USA). A Simplicity 185 apparatus (Millipore from Molsheim, France) generated the required ultrapure water (18.2 M $\Omega$ $\mathrm{cm})$.

A standard solution was prepared for each pesticide $(10,000 \mu \mathrm{g} / \mathrm{L}$ in $n$-hexane) and stored at $-18{ }^{\circ} \mathrm{C}$. These stocks were used to prepare standard mixture solutions of different concentrations in $n$-hexane. The mixture solutions were used as spiking levels $(50 \mathrm{ng} / \mathrm{g}$ dry weight (dw), $100 \mathrm{ng} / \mathrm{g} \mathrm{dw}, 200 \mathrm{ng} / \mathrm{g} \mathrm{dw}$ ), calibration curves and control solutions. Calibration curves were prepared from matrix-matching calibration solutions (between 
20 and $300 \mathrm{ng} / \mathrm{g}$ of $\mathrm{dw}$ ). The IS solution (100 $\mathrm{ng} / \mathrm{g} \mathrm{dw}$ concentration in $n$-hexane) was added to all experiments.

\section{Extraction procedure}

After lyophilisation, sediment samples were sieved using a 2-mm sieve (i.e. including granule, sand, silt and clay sediment size classes, which represent more than $50 \%$ of the sediment at all sampling sites; Rodrigues et al., submitted). Then, the material with a grain size $\leq 2 \mathrm{~mm}$ in diameter was ground in a grinder (Ultra Centrifugal Mill ZM 200) in order to obtain a homogeneous sample with grain sizes $\leq 0.25 \mathrm{~mm}$. Subsequently, pesticides were extracted and analysed. The extraction procedure was carried out according to Fernandes et al. (2013). Each sediment sample (5 g) was placed into a 50-mL centrifuge polypropylene tube. For validation studies, spikes $(50 \mu \mathrm{L}, 100 \mu \mathrm{L}$ and $200 \mu \mathrm{L})$ were made using working standard solutions, as to yield the necessary analyte concentrations. The IS solution $(10 \mu \mathrm{L}$ ) was added to all reagent blanks and samples (to yield $100 \mathrm{ng} / \mathrm{g} \mathrm{dw}$ ). An aliquot of the upper layer from each tube was placed into a vial and concentrated just to dryness using a gentle nitrogen stream. The residue obtained was reconstituted in $n$ hexane. The sample was subsequently capped, vortexed and then analysed in gas chromatography (GC).

\section{Analysis by gas chromatography-flame photometric detector}

Organophosphorus analysis was done with the Shimadzu GC-2010, equipped with a gas chromatography-flame photometric detector (GC-FPD) and a phosphorus filter. A ZBXLB capillary column (Zebron, Phenomenex) of $30 \mathrm{~m}$ with $0.25 \mu \mathrm{m}$ film thickness and $0.25 \mathrm{~mm}$ internal diameter was used. The seven pesticides were separated using the following oven programme in the GC: $50{ }^{\circ} \mathrm{C}$ with a 1-min holding time period, ramped at $10{ }^{\circ} \mathrm{C} / \mathrm{min}$ to $140{ }^{\circ} \mathrm{C}$ with a 1 -min holding time period, ramped at $5{ }^{\circ} \mathrm{C} / \mathrm{min}$ to $180{ }^{\circ} \mathrm{C}$ with a 2-min holding time period and finally ramped at $5{ }^{\circ} \mathrm{C} / \mathrm{min}$ to $270{ }^{\circ} \mathrm{C}$ with a 5 -min holding time period. The injector was at splitless mode $\left(250^{\circ} \mathrm{C}\right)$; the detection was done at $290{ }^{\circ} \mathrm{C}$. The carrier gas was helium (Linde Sogás); a constant $(1 \mathrm{~mL} / \mathrm{min})$ flow rate was used.

\section{Determination of sediment trace elements by X-ray fluorescence}

Sediment samples for trace element analysis were collected in late spring from different points in each study site. These samples were used to prepare a composite sample representative of each site. In laboratory, sediment samples were sieved with a 2-mmdiameter (most representative fraction at all sites) sieve and dried in an oven at $65{ }^{\circ} \mathrm{C}$ for about $24 \mathrm{~h}$. The dry samples were then milled to a particle size of $\leq 0.25 \mathrm{~mm}$ diameter and homogenised, in order to obtain fine-grained and homogeneous samples. The samples were put into the standard cell of an Instru-Med with the aid of a stainless steel spatula. For inversion of the samples' cup, the soil was hold firmly, with the aid of a paper disc, against the upper Polypropylene X-Ray Film from Premier Lab Supply (TF-240-255, 2.5 $\mathrm{cm}$ diameter and $4 \mu \mathrm{m}$ gauge).

A Niton® XL3t X-ray fluorescence (XRF) analyser was used. The instrument had a highperformance Si PIN diode detector equipped with a silver anode X-ray miniature tube; the excitation potential was up to $40 \mathrm{~kW}$. The mining mode was selected for the analysis. A qualitative analysis of 32 trace elements was done: silver (Ag), arsenic (As), gold ( $\mathrm{Au})$, 
barium $(\mathrm{Ba})$, calcium $(\mathrm{Ca})$, cadmium $(\mathrm{Cd})$, cobalt $(\mathrm{Co})$, chromium $(\mathrm{Cr})$, cesium $(\mathrm{Cs})$, copper $(\mathrm{Cu})$, iron $(\mathrm{Fe})$, mercury $(\mathrm{Hg})$, potassium $(\mathrm{K})$, manganese $(\mathrm{Mn})$, molybdenum (Mo), nickel $(\mathrm{Ni})$, lead $(\mathrm{Pb})$, palladium $(\mathrm{Pd})$, rubidium $(\mathrm{Rb})$, sulphur $(\mathrm{S})$, antimony $(\mathrm{Sb})$, scandium $(\mathrm{Sc})$, tin $(\mathrm{Sn})$, strontium $(\mathrm{Sr})$, tellurium $(\mathrm{Te})$, titanium $(\mathrm{Ti})$, thorium $(\mathrm{Th})$, uranium $(\mathrm{U})$, vanadium $(\mathrm{V})$, tungsten $(\mathrm{W})$, zinc $(\mathrm{Zn})$ and zirconium $(\mathrm{Zr})$. In the hydrographic basin of the AR, the main sector of activity is agriculture, and in the hydrographic basin of the FR, industrial activities (e.g. metallurgical industries, furniture polishing factories, hardware, mechanical locksmithing, slit-cut exploitation) are largely dominant, followed by agriculture, presenting more potential sources of trace metal pollution. XRF was used to measure elements in the sediments, and the results were shown in percentage $(\%)$.

\section{Biomarkers}

\section{Sampling and storage of macroinvertebrates}

Different tolerant and sensitive benthic macroinvertebrate taxa were selected for biomarker analysis, based on their presence in most sites of both rivers (according to preliminary data obtained in the spring of 2013). Organisms were seasonally sampled (autumn, in November 2013; spring, in June 2014; summer, in September 2014) at each sampling site of the AR and FR (Table 1). For both rivers, despite increased sampling effort, not all taxa could be found in all of the studied seasons. Sampling of the biological material (using a 500- $\mu \mathrm{m}$-mesh hand net) took place in different organic (macrophytes and coarse particulate organic matter) and inorganic (blocks, stones, gravel and sand + silt + clay) substrates that covered the river bed. Organisms were placed in plastic vessels filled with river water and immediately taken to the laboratory. Each taxonomic group (Table 1) was sorted out alive, directly from the plastic containers, with tweezers for Odonata and Trichoptera and plastic pipettes with tips of different sizes for Diptera and Ephemeroptera. They were identified morphologically up to the family or genus level (Tachet et al. 2002) using a magnifying glass. For some taxa, identification up to the genus level was not possible (Simuliidae, Chironomidae and Polycentropodidae families) or would be very time-consuming (requiring a microscope to see morphological details) and stressful for the organisms. Five to one hundred benthic macroinvertebrates of each taxon, depending on the sizes of the organisms (five each of Trichoptera: Hydropsyche spp. and Polycentropodidae; Odonata: Gomphus spp., Boyeria spp. and Calopteryx spp.; Hemiptera: Nepa spp.; 20 each of Ephemeroptera: Baetis spp., Caenis spp., Abrophlebia spp. and Ephemerella spp.; 50 and 100 each of Diptera: Simuliidae and Chironomidae, respectively; organisms of the same taxon were of similar size), were placed in different microtubes and promptly frozen in liquid nitrogen. Microtubes were then preserved at $-80{ }^{\circ} \mathrm{C}$ until biomarker analysis. Before this procedure, preliminary laboratory tests were made to optimise the removal of organisms from the containers, so as to minimise stress to organisms, as well as to determine the number of organisms of the same taxa to be integrated in each pool, in order to ensure the amount of biomass needed to record enzymatic activity.

Table 1 Benthic macroinvertebrate taxa (genus or family) sampled for biomarker analysis in the Âncora and the Ferreira rivers in each season 


\begin{tabular}{|l|l|l|}
\hline Seasons & Âncora River & Ferreira River \\
\hline Autumn & Baetidae (Baetis spp.) & Baetidae (Baetis spp.) \\
\hline & Calopterygidae (Calopteryx spp.) & Calopterygidae (Calopteryx spp.) \\
\hline & Aeshnidae (Boyeria spp.) & Chironomidae \\
\hline Spring & Gomphidae (Gomphus spp.) & \\
\hline & Baetidae (Baetis spp.) & Baetidae (Baetis spp.) \\
\hline Calopterygidae (Calopteryx spp.) & Calopterygidae (Calopteryx spp.) \\
\hline Aeshnidae (Boyeria spp.) & Aeshnidae (Boyeria spp.) \\
\hline Chironomidae & Chironomidae \\
\hline & Gomphidae (Gomphus spp.) & Hydropsychidae (Hydropsyche spp.) \\
\hline & Leptophlebiidae (Abrophlebia spp.) & Nepidae (Nepa spp.) \\
\hline Ephemerellidae (Ephemerella spp.) & Caenidae (Caenis spp.) \\
\hline & Polycentropodidae & Simuliidae \\
\hline Baetidae (Baetis spp.) & Baetidae (Baetis spp.) \\
\hline Calopterygidae (Calopteryx spp.) & Calopterygidae (Calopteryx spp.) \\
\hline Aeshnidae (Boyeria spp.) & Aeshnidae (Boyeria spp.) \\
\hline Chironomidae & Chironomidae \\
\hline Gomphidae (Gomphus spp.) & Hydropsychidae (Hydropsyche spp.) \\
\hline Leptophlebiidae (Abrophlebia spp.) & Nepidae (Nepa spp.) \\
\hline Ephemerellidae (Ephemerella spp.) & Caenidae (Caenis spp.) \\
\hline
\end{tabular}

\section{Biomarker analysis}

In this study, as well as in most studies using insect larvae (e.g. Berra et al. 2004; Barata et al. 2005; Bonzini et al. 2008; Minutoli et al. 2013), the analysis of all biomarkers was performed in triplicate pools (three independent replicates for each taxon using the whole body of the organisms), per study site and taxon. For the measurement of lactate dehydrogenase (LDH) activity, the microtubes first underwent three frozen/unfrozen cycles (at $-20{ }^{\circ} \mathrm{C}$ and room temperature). Their content was then homogenised in icecold Tris/ $\mathrm{NaCl}$ buffer ( $\mathrm{pH} 7.2$ ), followed by centrifugation at $3300 \times g$ and $4{ }^{\circ} \mathrm{C}$, for $3 \mathrm{~min}$, as described by Diamantino et al. (2001). The resulting supernatants were used to assess the enzyme activity by the method of Vassault (1983), in a microplate reader. Briefly, the method measures the pyruvate consumed due to NADH oxidation; the reaction is followed at $340 \mathrm{~nm}$ wavelength. For the determination of cholinesterase (ChE) activity, homogenisation was done in ice-cold phosphate buffer (0.1 M; $\mathrm{pH} 7.2)$ and centrifugation was carried out at $3300 \times \mathrm{g}$ and $4{ }^{\circ} \mathrm{C}$ for $3 \mathrm{~min}$ (Guilhermino et al. 1996). ChE activity was measured in the supernatant by Ellman's method (Ellman et al. 1961). Briefly, this method consists in measuring the rate of production of thiocholine, as acetylthiocholine is hydrolysed by enzymatic action. Thiocholine complexes with 5,5'-dithiobis(2nitrobenzoic acid) (DTNB), leading to the formation of a yellow compound, whose increase can be followed at $412 \mathrm{~nm}$ in a microplate reader. The substrate used in all the assays was acetylthiocholine, measuring the whole activity produced by the forms of ChEs that may be present in the samples (Guilhermino et al. 1996). For the determination of antioxidant enzymes (catalase (CAT), glutathione $S$-transferase (GST)) and the levels of lipid peroxidation (LPO), the homogenisation was done in phosphate buffer (with $0.1 \%$ Triton X-100; $50 \mathrm{mM} ; \mathrm{pH} 7.0$ ) and the centrifugation took place at $15,000 \times g$ and $4{ }^{\circ} \mathrm{C}$ for $10 \mathrm{~min}$. Aliquots of the supernatant were used to determine each biomarker (GST, CAT and LPO). The activity of GST enzyme was measured following the method of Habig et al. (1974). The reaction is based on the conjugation of reduced glutathione (GSH) with 1-chloro-2,4-dinitrobenzene (CDNB), which can be measured in a microplate (Frasco and Guilhermino 2002) at a $340 \mathrm{~nm}$ wavelength. The measurement of CAT activity was done by following the consumption of $\mathrm{H}_{2} \mathrm{O}_{2}$ in a spectrophotometer at 240 $\mathrm{nm}$, according to Aebi (1984). The extent of LPO was assayed through the quantification of the amount of thiobarbituric acid-reactive substances (TBARS) at $535 \mathrm{~nm}$, following the method of Buege and Aust (1978). Briefly this method is based on the reaction of the 
lipid peroxidation by-products, as malondialdehyde (MDA), with the 2-thiobarbituric acid (TBA). Catalase activity is presented in micromoles/minute/milligram of protein. All other enzyme activities are presented in nanomoles/minute/milligram of protein. The amount of TBARS is presented in nanomoles of MDA equivalents/milligram of protein. All measurements were done in triplicate except for LPO which was measured in duplicate. The concentration of protein in the samples was quantified with the method of Bradford (1976), also using a microplate reader, at a wavelength of $600 \mathrm{~nm}$, and using as standard bovine $\gamma$-globulins purchased from Sigma-Aldrich (USA). All microplate determinations were carried out in a Thermo Scientific ${ }^{\mathrm{TM}}$ Multiskan GO Microplate Spectrophotometer. CAT activities were assessed in a UV/Vis spectrophotometer (UV$3100 \mathrm{PC}, \mathrm{VWR})$. All enzymatic activities, and respective protein measurements, were done at $25{ }^{\circ} \mathrm{C}$.

\section{Statistical data analysis}

The spatial variation of biomarker responses in the different macroinvertebrate families in each season and river was visualised in Tukey boxplots, showing data distribution, medians and quartiles (Chambers et al. 1983).

Since most of the variables analysed in this study failed the test of Shapiro-Wilk for normality and/or the test of Levene for homogeneity of variance across groups, we opted for non-parametric statistical testing. Differences between rivers in each season regarding environmental (water physico-chemical parameters, hydromorphological indices and sediment parameters, i.e. percentage of metals and concentration of pesticides) and biological (community index and biomarker responses in the taxa that were common to both rivers, i.e. Chironomidae, Baetis spp., Calopteryx spp. and Boyeria spp.) parameters were assessed with the Kruskal-Wallis test. A pairwise post hoc analysis was done with the Wilcoxon rank sum test, corrected for the familywise error rate, to detect which water physico-chemical parameters and hydromorphological and biological indices differed among seasons or among sites within each river (Hollander and Wolfe 1973). For all statistical tests performed, a significance level of 0.05 was considered.

Multivariate analysis was used for a better assessment of biomarker applicability for diagnostic purposes. To minimise problems of missing values, high dimensionality and difficulty in obtaining complete data for some taxa (resulting from their high sensitivity to environmental quality), multivariate analysis was done using only data from the more widespread, abundant or bigger-sized (requiring less organisms for the biomarker determinations) macroinvertebrate taxa, i.e. Calopteryx spp., Baetis spp. and Chironomidae. Furthermore, multivariate analysis was restricted to spring and summer data, because these were the seasons for which all biomarkers could be determined at all sampling sites in both rivers. First, a detrended correspondence analysis (DCA) was applied to determine the relative length of the data gradient. This was used to decide on the suitable data analysis technique (i.e. unimodal or linear response). Then and since all of our DCA gradient lengths were $<2$, a principal component analysis (PCA) was employed to characterise the main patterns of taxon-related, spatial and seasonal variation in biomarker responses (according to Ter Braak and Prentice 1988). To assess response profiles of the three taxa, PCA was done first using biomarker levels and the macroinvertebrate index as quantitative variables, taxa and season (spring and summer) as qualitative supplementary variables and water physico-chemical parameters and hydromorphological indices as supplementary quantitative variables. Following the 
principal components (PCs) extracted and the analysis of the cloud of individuals, subsequent PCAs were carried out for each taxon individually, with river and season as supplementary qualitative variables.

Boxplots, statistical tests and multivariate analyses were done with the Statistical programme R, package version 3.4.2, including the Rcmdr and FactoMineR packages (Le et al. 2008; R Core Team 2017; Fox and Bouchet-Valat 2018).

\section{Results}

\section{Physico-chemical parameters}

Significant differences between the AR and FR $(p<0.05)$ were found for dissolved oxygen (concentration, DO; \% saturation, \% DO) in summer, ammonium ion in autumn and water temperature, nitrates, nitrites, conductivity, salinity, total dissolved and suspended solids in all seasons (Table 2). The FR reached higher values than the AR for most parameters, except for water temperature in autumn and summer, and dissolved oxygen (DO and \% DO) in summer. Significant seasonal differences were found for water temperature in both rivers, for COD, nitrates and total phosphorus in the AR and for dissolved oxygen (DO and \% DO) and nitrites in the FR (Table 2 ). In both rivers, all physico-chemical parameters, except nitrites in the AR and water temperature, DO and $\%$ DO in the FR, differed significantly among sites (Table 2 ).

Table 2 Spatial and seasonal variation of water physico-chemical parameters determined in the Âncora River (AR) and Ferreira River (FR)

\begin{tabular}{|c|c|c|c|c|c|c|c|c|c|c|c|c|c|c|c|c|c|}
\hline & \multicolumn{8}{|l|}{ AR } & \multicolumn{9}{|l|}{ FR } \\
\hline & \multicolumn{5}{|c|}{ Sampling site } & \multicolumn{3}{|l|}{ Season } & \multicolumn{6}{|c|}{ Sampling site } & \multicolumn{3}{|l|}{ Season } \\
\hline & AR1 & AR2 & AR3 & AR4 & AR5 & Autumn & Spring & Summer & FR1 & FR2 & FR3 & FR4 & FR5 & FR6 & Autumn & Spring & Summer \\
\hline Temp & $\begin{array}{l}14.46 \pm \\
2.38^{\mathrm{a}} \pm\end{array}$ & $\begin{array}{l}15.38 \\
\pm \\
2.16^{\mathrm{ab}}\end{array}$ & $\begin{array}{l}15.57 \\
\pm \\
1.99^{\mathrm{bc}}\end{array}$ & $\begin{array}{l}16.39 \\
\pm \\
3.20^{b c}\end{array}$ & $\begin{array}{l}16.19 \\
\pm \\
2.90^{c}\end{array}$ & $\begin{array}{l}12.75 \pm \\
0.51^{\mathrm{a}} \pm\end{array}$ & $\begin{array}{l}16.78 \\
\pm 1.43^{b}\end{array}$ & $\begin{array}{l}17.26 \pm \\
0.78^{\mathrm{b}}\end{array}$ & $\begin{array}{l}15.92 \pm \\
4.95\end{array}$ & $\begin{array}{l}15.48 \pm \\
4.26\end{array}$ & $\begin{array}{l}15.97 \pm \\
5.32\end{array}$ & $\begin{array}{l}15.60 \pm \\
4.86\end{array}$ & $\begin{array}{l}15.33 \pm \\
4.84\end{array}$ & $\begin{array}{l}15.30 \\
\pm 5.32\end{array}$ & $\begin{array}{l}0.46 \pm \\
49^{\mathrm{a}} \pm\end{array}$ & $\begin{array}{l}20.25 \pm \\
0.71^{\mathrm{b}} \pm\end{array}$ & $\begin{array}{l}16.09 \pm \\
0.09^{c}\end{array}$ \\
\hline $\mathrm{pH}$ & $\begin{array}{l}5.98 \pm \\
1.24^{\mathrm{ab}}\end{array}$ & $\begin{array}{l}5.32 \pm \\
0.16^{\mathrm{b}}\end{array}$ & $\begin{array}{l}5.73 \pm \\
0.22^{\mathrm{a}}\end{array}$ & $\begin{array}{l}5.82 \pm \\
0.16^{\mathrm{a}}\end{array}$ & $\begin{array}{l}5.81 \pm \\
0.16^{\mathrm{a}}\end{array}$ & $\begin{array}{l}5.97 \pm \\
0.85\end{array}$ & & & $\begin{array}{l}6.46 \pm \\
0.48^{\mathrm{abc}}\end{array}$ & $\begin{array}{l}6.05 \pm \\
0.17^{\text {ad }}\end{array}$ & $\begin{array}{l}6.50 \pm \\
0.47^{\mathrm{abd}}\end{array}$ & $\begin{array}{l}6.70 \pm \\
0.39^{\mathrm{bc}}\end{array}$ & $\begin{array}{l}6.75 \pm \\
0.26^{\mathrm{c}}\end{array}$ & $\begin{array}{l}6.30 \pm \\
1.89^{\mathrm{d}}\end{array}$ & $\begin{array}{l}6.38 \pm \\
0.23\end{array}$ & $\begin{array}{l}6.52 \pm \\
0.30\end{array}$ & $\begin{array}{l}6.66 \pm \\
0.32\end{array}$ \\
\hline DO & $\begin{array}{l}9.03 \pm \\
0.49^{\mathrm{a}}\end{array}$ & $\begin{array}{l}9.11 \pm \\
0.81^{\mathrm{a}}\end{array}$ & $\begin{array}{l}7.34 \pm \\
1.76^{\mathrm{b}}\end{array}$ & $\begin{array}{l}7.39 \pm \\
2.75^{\mathrm{ab}}\end{array}$ & $\begin{array}{l}8.03 \pm \\
2.67^{\mathrm{ab}}\end{array}$ & $\begin{array}{l}9.42 \pm \\
1.06\end{array}$ & $\begin{array}{l}6.46 \pm \\
1.97\end{array}$ & $\begin{array}{l}8.66 \pm \\
0.81\end{array}$ & $\begin{array}{l}7.17 \pm \\
1.76\end{array}$ & $\begin{array}{l}7.29 \pm \\
1.86\end{array}$ & $\begin{array}{l}7.32 \pm \\
1.61\end{array}$ & $\begin{array}{l}6.87 \pm \\
1.12\end{array}$ & $\begin{array}{l}7.47 \pm \\
1.86\end{array}$ & $\begin{array}{l}7.09 \pm \\
1.67\end{array}$ & $\begin{array}{l}8.34 \pm \\
0.41^{\mathrm{a}}\end{array}$ & $\begin{array}{l}7.92 \pm \\
0.51^{\mathrm{a}}\end{array}$ & $\begin{array}{l}5.35 \pm \\
0.18^{\mathrm{b}}\end{array}$ \\
\hline $\begin{array}{l}\% \\
\text { DO }\end{array}$ & $\begin{array}{l}90.67 \pm \\
0.68^{\mathrm{abc}}\end{array}$ & $\begin{array}{l}90.97 \\
\pm \\
4.99 \mathrm{bc}\end{array}$ & $\begin{array}{l}79.13 \\
\pm \\
11.84^{\mathrm{a}}\end{array}$ & $\begin{array}{l}82.50 \\
\pm \\
11.53^{b}\end{array}$ & $\begin{array}{l}91.87 \\
\pm \\
7.83^{c}\end{array}$ & $\begin{array}{l}89.04 \pm \\
9.69\end{array}$ & $\begin{array}{l}81.34 \\
\pm 7.19\end{array}$ & $\begin{array}{l}90.7 \pm \\
8.55\end{array}$ & $\begin{array}{l}73.87 \pm \\
19.90\end{array}$ & $\begin{array}{l}74.43 \pm \\
19.34\end{array}$ & $\begin{array}{l}74.03 \pm \\
17.07\end{array}$ & $\begin{array}{l}68.47 \pm \\
10.96\end{array}$ & $\begin{array}{l}73.80 \pm \\
17.64\end{array}$ & $\begin{array}{l}69.63 \\
\pm \\
13.34\end{array}$ & $\begin{array}{l}74.92 \pm \\
3.24^{\mathrm{a}}\end{array}$ & $\begin{array}{l}87.22 \pm \\
6.43^{b}\end{array}$ & $\begin{array}{l}54.98 \pm \\
1.35^{c}\end{array}$ \\
\hline COD & $\begin{array}{l}8.00 \pm \\
13.00^{\mathrm{ab}}\end{array}$ & $\begin{array}{l}4.67 \pm \\
7.23^{\mathrm{ab}}\end{array}$ & $\begin{array}{l}1.00 \pm \\
1.73^{\mathrm{a}}\end{array}$ & $\begin{array}{l}5.00 \pm \\
7.81^{\mathrm{ab}}\end{array}$ & $\begin{array}{l}5.33 \pm \\
5.03^{\mathrm{b}}\end{array}$ & $\begin{array}{l}1.20 \pm \\
2.68^{\mathrm{b}}\end{array}$ & $\begin{array}{l}12.60 \\
\pm 7.23^{\mathrm{a}} \\
\end{array}$ & $\begin{array}{l}0.60 \pm \\
0.55^{\mathrm{b}}\end{array}$ & $67 \pm$ & $\begin{array}{l}17.00 \pm \\
18.68^{\mathrm{a}}\end{array}$ & $\begin{array}{l}0.00 \pm \\
0.00^{\mathrm{b}}\end{array}$ & $\begin{array}{l}15.00 \pm \\
24.27^{\mathrm{a}}\end{array}$ & $\begin{array}{l}0.00 \pm \\
0.00^{\mathrm{b}}\end{array}$ & $\begin{array}{l}5.00 \pm \\
8.66^{\mathrm{a}}\end{array}$ & $67 \pm$ & $\begin{array}{l}9.33 \pm \\
15.10\end{array}$ & $\begin{array}{l}2.33 \pm \\
5.72\end{array}$ \\
\hline Cond & $\begin{array}{l}21.00 \pm \\
3.61^{\mathrm{a}} \pm\end{array}$ & $\begin{array}{l}35.67 \\
\pm \\
2.31^{b}\end{array}$ & $\begin{array}{l}39.67 \\
\pm 3.21^{\mathrm{c}}\end{array}$ & $\begin{array}{l}44.00 \\
\pm 6.93^{\mathrm{c}}\end{array}$ & $\begin{array}{l}45.00 \\
\pm \\
2.65^{d}\end{array}$ & $\begin{array}{l}37.4 \pm \\
7.37\end{array}$ & $\begin{array}{l}37.4 \pm \\
10.53\end{array}$ & $\begin{array}{l}36.4 \pm \\
12.62\end{array}$ & $\begin{array}{l}118.33 \\
\pm \\
12.74^{\mathrm{a}}\end{array}$ & $\begin{array}{l}124.33 \\
\pm \\
21.13^{\mathrm{a}}\end{array}$ & $\begin{array}{l}148.00 \\
\pm \\
26.89^{b}\end{array}$ & $\begin{array}{l}167.67 \\
\pm \\
14.29^{c}\end{array}$ & $\begin{array}{l}183.00 \\
\pm \\
10.44^{d}\end{array}$ & $\begin{array}{l}172.33 \\
\pm 8.02^{c}\end{array}$ & $\begin{array}{l}150.83 \\
\pm 31.47\end{array}$ & $\begin{array}{l}160.33 \\
\pm 34.75\end{array}$ & $\begin{array}{l}145.67 \pm \\
221.77\end{array}$ \\
\hline Sal & $\begin{array}{l}0.01 \pm \\
0.00^{\mathrm{a}}\end{array}$ & $\begin{array}{l}0.02 \pm \\
0.01^{\mathrm{b}}\end{array}$ & $\begin{array}{l}0.02 \pm \\
0.00^{\mathrm{b}}\end{array}$ & $\begin{array}{l}0.02 \pm \\
0.00^{\mathrm{b}}\end{array}$ & $\begin{array}{l}0.02 \pm \\
0.00^{\mathrm{b}}\end{array}$ & $\begin{array}{l}0.02 \pm \\
0.00\end{array}$ & & & $\begin{array}{l}0.06 \pm \\
0.01^{\mathrm{a}}\end{array}$ & $\begin{array}{l}0.06 \pm \\
0.01^{\mathrm{a}}\end{array}$ & $\begin{array}{l}0.07 \pm \\
0.01^{\mathrm{b}}\end{array}$ & $\begin{array}{l}0.08 \pm \\
0.01^{\mathrm{c}}\end{array}$ & $\begin{array}{l}0.09 \pm \\
0.01^{\mathrm{d}}\end{array}$ & $\begin{array}{l}0.08 \pm \\
0.00^{e}\end{array}$ & $\begin{array}{l}0.07 \pm \\
0.01\end{array}$ & $\begin{array}{l}0.07 \pm \\
0.02\end{array}$ & $\begin{array}{l}0.07 \pm \\
0.01\end{array}$ \\
\hline TDS & $\begin{array}{l}10.33 \pm \\
1.53^{\mathrm{a}}\end{array}$ & $\begin{array}{l}17.67 \\
\pm \\
1.53^{b}\end{array}$ & $\begin{array}{l}19.67 \\
\pm 1.53^{c}\end{array}$ & $\begin{array}{l}22.00 \\
\pm \\
3.46^{d}\end{array}$ & $\begin{array}{l}22.67 \\
\pm \\
1.15^{\mathrm{e}}\end{array}$ & $\begin{array}{l}18.6 \pm \\
3.85\end{array}$ & $\begin{array}{l}18.6 \pm \\
5.27\end{array}$ & $\begin{array}{l}18.2 \pm \\
6.42\end{array}$ & $\begin{array}{l}59.00 \pm \\
6.08^{\mathrm{a}}\end{array}$ & $\begin{array}{l}62.00 \pm \\
10.54^{\mathrm{a}}\end{array}$ & $\begin{array}{l}74.33 \pm \\
13.58^{\mathrm{b}}\end{array}$ & $\begin{array}{l}83.67 \pm \\
7.09^{c}\end{array}$ & $\begin{array}{l}91.33 \pm \\
5.51^{\mathrm{d}}\end{array}$ & $\begin{array}{l}86.00 \\
\pm 4.00^{c}\end{array}$ & $\begin{array}{l}75.17 \pm \\
15.68\end{array}$ & $\begin{array}{l}80.17 \pm \\
17.51\end{array}$ & $\begin{array}{l}72.83 \pm \\
10.70\end{array}$ \\
\hline TSS & $\begin{array}{l}1.61 \pm \\
1.56^{\mathrm{ab}}\end{array}$ & $\begin{array}{l}1.74 \pm \\
0.64^{\mathrm{a}}\end{array}$ & $\begin{array}{l}1.24 \pm \\
1.41^{\mathrm{a}}\end{array}$ & $\begin{array}{l}1.34 \pm \\
1.27^{\mathrm{a}}\end{array}$ & $\begin{array}{l}1.78 \pm \\
1.77^{\mathrm{a}}\end{array}$ & $\begin{array}{l}0.81 \pm \\
0.54\end{array}$ & $\begin{array}{l}2.61 \pm \\
1.25\end{array}$ & $\begin{array}{l}1.2 \pm \\
0.92\end{array}$ & $\begin{array}{l}1.43 \pm \\
1.53^{\mathrm{a}}\end{array}$ & $\begin{array}{l}5.86 \pm \\
4.96^{\mathrm{bc}}\end{array}$ & $\begin{array}{l}3.60 \pm \\
4.32^{\mathrm{ab}}\end{array}$ & $\begin{array}{l}3.89 \pm \\
3.38^{\mathrm{bc}}\end{array}$ & $\begin{array}{l}3.97 \pm \\
3.20^{\mathrm{bc}}\end{array}$ & $\begin{array}{l}6.03 \pm \\
4.01^{\mathrm{c}}\end{array}$ & $\begin{array}{l}1.26 \pm \\
1.09\end{array}$ & $\begin{array}{l}6.52 \pm \\
3.54\end{array}$ & $\begin{array}{l}4.62 \pm \\
3.34\end{array}$ \\
\hline $\mathrm{P}$ & $\begin{array}{l}0.19 \pm \\
0.17^{\mathrm{a}}\end{array}$ & $\begin{array}{l}0.11 \pm \\
0.08^{\mathrm{b}}\end{array}$ & $\begin{array}{l}0.12 \pm \\
0.08^{\mathrm{b}}\end{array}$ & $\begin{array}{l}0.26 \pm \\
0.22^{\mathrm{a}}\end{array}$ & $\begin{array}{l}0.10 \pm \\
0.09^{\mathrm{b}}\end{array}$ & $\begin{array}{l}0.05 \pm \\
0.02^{\mathrm{a}}\end{array}$ & $\begin{array}{l}0.29 \pm \\
0.14^{\mathrm{b}}\end{array}$ & $\begin{array}{l}0.12 \pm \\
0.06^{\mathrm{c}}\end{array}$ & $\begin{array}{l}0.12 \pm \\
0.11^{\mathrm{a}}\end{array}$ & $\begin{array}{l}0.27 \pm \\
0.17^{\mathrm{b}}\end{array}$ & $\begin{array}{l}0.42 \pm \\
0.20^{c}\end{array}$ & $\begin{array}{l}0.82 \pm \\
0.46^{d}\end{array}$ & $\begin{array}{l}0.83 \pm \\
0.55^{\mathrm{d}}\end{array}$ & $\begin{array}{l}0.61 \pm \\
0.29^{d}\end{array}$ & $\begin{array}{l}0.25 \pm \\
0.13\end{array}$ & $\begin{array}{l}0.56 \pm \\
0.21\end{array}$ & $\begin{array}{l}0.73 \pm \\
0.57\end{array}$ \\
\hline $\mathrm{NO}_{3}^{-}$ & $\begin{array}{l}7.09 \pm \\
6.95^{\mathrm{ac}}\end{array}$ & $\begin{array}{l}8.76 \pm \\
2.72^{\mathrm{ac}}\end{array}$ & $\begin{array}{l}14.13 \\
\pm .5 \\
7.58^{\mathrm{b}}\end{array}$ & $\begin{array}{l}10.09 \\
\pm \\
1.70^{\mathrm{ab}}\end{array}$ & $\begin{array}{l}8.37 \pm \\
2.10^{\circ}\end{array}$ & $\begin{array}{l}10.45 \pm \\
7.11^{\mathrm{ab}}\end{array}$ & $\begin{array}{l}6.53 \pm \\
2.85^{\mathrm{a}}\end{array}$ & $\begin{array}{l}12.08 \pm \\
1.74^{\mathrm{b}} \pm\end{array}$ & $\begin{array}{l}22.30 \pm \\
5.95^{\mathrm{ac}}\end{array}$ & $\begin{array}{l}19.34 \pm \\
7.09^{\mathrm{a}}\end{array}$ & $\begin{array}{l}25.20 \pm \\
8.49^{\mathrm{ac}}\end{array}$ & $\begin{array}{l}27.07 \pm \\
2.15^{\mathrm{bc}}\end{array}$ & $\begin{array}{l}31.35 \pm \\
7.57^{b}\end{array}$ & $\begin{array}{l}27.17 \\
\pm \\
10.39^{c}\end{array}$ & $\begin{array}{l}25.82 \pm \\
2.99\end{array}$ & $\begin{array}{l}21.76 \pm \\
4.09\end{array}$ & $\begin{array}{l}28.65 \pm \\
11.37\end{array}$ \\
\hline $\mathrm{NO}_{2}^{-}$ & $\begin{array}{l}0.00 \pm \\
0.00\end{array}$ & $\begin{array}{l}0.00 \pm \\
0.00\end{array}$ & $\begin{array}{l}0.00 \pm \\
0.00\end{array}$ & $\begin{array}{l}0.00 \pm \\
0.00\end{array}$ & $\begin{array}{l}0.00 \pm \\
0.00\end{array}$ & $\begin{array}{l}0.00 \pm \\
0.00\end{array}$ & $\begin{array}{l}0.00 \pm \\
0.00\end{array}$ & $\begin{array}{l}0.00 \pm \\
0.00\end{array}$ & $\begin{array}{l}2.56 \pm \\
4.43^{\mathrm{a}}\end{array}$ & $\begin{array}{l}4.34 \pm \\
7.50^{\mathrm{a}}\end{array}$ & $\begin{array}{l}5.69 \pm \pm \\
9.51^{\mathrm{b}}\end{array}$ & $\begin{array}{l}8.93 \pm \\
13.93^{c}\end{array}$ & $\begin{array}{l}13.03 \pm \\
20.21^{\mathrm{c}}\end{array}$ & $\begin{array}{l}10.11 \\
\pm \\
16.07^{c}\end{array}$ & $\begin{array}{l}0.29 \pm \\
0.22^{\mathrm{a}}\end{array}$ & $\begin{array}{l}21.22 \mathrm{t}^{\mathrm{t}} \\
10.68^{\mathrm{b}}\end{array}$ & $\begin{array}{l}0.81 \pm \\
0.96^{\mathrm{a}}\end{array}$ \\
\hline $\mathrm{NH}_{4}^{+}$ & $\begin{array}{l}0.59 \pm \\
0.18^{\mathrm{a}}\end{array}$ & $\begin{array}{l}0.47 \pm \\
0.14^{\mathrm{b}}\end{array}$ & $\begin{array}{l}0.42 \pm \\
0.13^{\mathrm{b}}\end{array}$ & $\begin{array}{l}0.32 \pm \\
0.05^{\mathrm{c}}\end{array}$ & $\begin{array}{l}0.27 \pm \\
0.21^{\mathrm{d}}\end{array}$ & $\begin{array}{l}0.43 \pm \\
0.07\end{array}$ & $\begin{array}{l}0.35 \pm \\
0.20\end{array}$ & $\begin{array}{l}0.46 \pm \\
0.24\end{array}$ & $\begin{array}{l}0.30 \pm \\
0.13^{\mathrm{a}}\end{array}$ & $\begin{array}{l}0.64 \pm \\
0.59^{\mathrm{b}}\end{array}$ & $\begin{array}{l}0.80 \pm \\
0.78^{\mathrm{be}}\end{array}$ & $\begin{array}{l}3.14 \pm \\
1.91^{\mathrm{c}}\end{array}$ & $\begin{array}{l}2.29 \pm \\
1.89^{\mathrm{d}}\end{array}$ & $\begin{array}{l}1.12 \pm \\
0.70^{\mathrm{e}}\end{array}$ & $\begin{array}{l}1.45 \pm \\
0.60\end{array}$ & $\begin{array}{l}0.64 \pm \\
0.69\end{array}$ & $\begin{array}{l}2.06 \pm \\
2.25\end{array}$ \\
\hline
\end{tabular}


Overall, DO and \% DO values were within the limit values for "good" ecological status of Northern Portuguese rivers (i.e. DO $\geq 5 \mathrm{mg} \mathrm{O}_{2} / \mathrm{L} ; \%$ DO $60-120 \%$ of saturation) in both studied rivers and in all seasons (Table 2), except for the \% DO at all FR sites in summer. In the FR, lower DO and \% DO means were observed in the summer compared to autumn and spring (Table $\underline{2}$ ).

In the AR, COD and phosphorus levels were significantly higher in spring compared to the remaining seasons (Table 2). High levels of phosphorus (i.e. $\mathrm{P}>0.10 \mathrm{mg} / \mathrm{L}$ ) were observed at all AR sites in spring, as well as in AR1, AR3 and AR4 in summer. Although nitrate values observed at all AR sites and seasons were considered "good" (i.e. $\mathrm{NO}_{3}{ }^{-}$ $\leq 25 \mathrm{mg} / \mathrm{L}$ ), spring values were significantly lower than those observed in autumn and summer (Table 2 ).

In the FR, high levels of nutrients (i.e. $\mathrm{P}>0.10 \mathrm{mg} / \mathrm{L} ; \mathrm{NO}_{3}{ }^{-}>25 \mathrm{mg} / \mathrm{L} ; \mathrm{NO}_{2}{ }^{-}>0.1 \mathrm{mg} / \mathrm{L}$; $\mathrm{NH}_{4}{ }^{+}>1 \mathrm{mg} / \mathrm{L}$ ) were found in all seasons, especially at the downstream sites (FR4 to FR6). Conductivity, salinity and TDS were also higher at the downstream sites compared to the upstream sites (Table 2). In the FR, the amount of nitrites differed significantly between seasons, with higher values occurring in spring compared to autumn and summer (Table 2).

\section{Biological and hydromorphological indices}

Scores of the biological ( $\operatorname{IPtI}_{\mathrm{N}}$ ) and hydromorphological (HQA and HMS) indices, determined in the same sites and seasons as the macroinvertebrate sampling campaigns for biomarker measurements, were obtained from Rodrigues et al. (submitted). According to the IPtI index, AR sites presented "good" or "high" ecological status in almost all studied seasons (AR3 and AR5 had "moderate" ecological status in autumn), while FR sites presented ecological status from "moderate" to "bad" (Rodrigues et al., submitted). Regarding the hydromorphological characterisation of the sampling sites, despite the HQA index revealing that almost all AR sites (except AR3 in summer) and some FR sites (FR1, FR3 and FR4) had high physical habitat heterogeneity ("high" ecological status), the HMS index indicated that, in general, channel morphology of both rivers was artificially modified (except at AR3).

Significant differences between rivers $(p<0.05)$ were found for all indices in the different seasons, except for HQA in the summer $(p=0.0541)$, with the AR reaching higher $\mathrm{IPtI}_{\mathrm{N}}$ and HQA scores and lower HMS scores than the FR (Table $\underline{6}$ in Appendix 2). In both rivers, significant differences between sites were found for the biological and hydromorphological indices determined, but not among seasons.

\section{Pesticides and trace elements in the sediment}

Calibration curves were performed for standards dissolved in solvent and for matrix standard extracts. Because of the presence of matrix interferences, a matrix-matched calibration curve was more appropriate for the quantification of such pesticides in the samples of sediments. For all pesticides, and over the range of concentrations tested, these were found to be linear for both studied rivers, as indicated by the very high values of the determination coefficient $\left(r^{2}>0.99\right)$ (Table $\underline{7}$ in Appendix 3). For all pesticides, limit of detection (LOD) and limit of quantification (LOQ) were in a range from 11.7 to $27.2 \mathrm{ng} / \mathrm{g}$ $\mathrm{dw}$ and from 39 to $90.8 \mathrm{ng} / \mathrm{g} \mathrm{dw}$, respectively. 
Recovery experiments were performed in duplicate, at three concentrations ( $50 \mathrm{ng} / \mathrm{g} \mathrm{dw}$, $100 \mathrm{ng} / \mathrm{g} \mathrm{dw}$ and $200 \mathrm{ng} / \mathrm{g} \mathrm{dw}$ ), for sediment with a grain size $\leq 0.25 \mathrm{~mm}$. The results obtained ranged between 71 and $118 \%$, with $2-10 \%$ relative standard deviation (RSD). The recovery experiments for sediments with larger grain sizes $(\leq 2 \mathrm{~mm})$ showed lower recovery percentages than expected at the level of $100 \mathrm{ng} / \mathrm{g} \mathrm{dw}$, mainly for dimethoate and chlorpyrifos. Finally, the ultrasonic bath followed by standard EN 15662 citrateQuEChERS procedure enabled better recoveries of pesticides with the extraction for sediment samples $\leq 0.25 \mathrm{~mm}$ grain size and spiked at $50 \mathrm{ng} / \mathrm{g} \mathrm{dw}$ (recoveries between 83 and 114\%; Table 7 in Appendix 3). All pesticide analyses were performed with sediment samples of $\leq 0.25 \mathrm{~mm}$ grain size, and only the presence of chlorpyrifos was detected in both rivers, at AR1 and AR3 from the AR and at FR1, FR2 and FR5 from the FR (Table 3).

Table 3 Chlorpyrifos concentration in the sediment of the sampling sites of the Âncora (AR1 and AR3) and Ferreira (FR1, FR2 and FR5) rivers where it was detected

\begin{tabular}{|l|l|}
\hline Sites & Chlorpyrifos $\mathbf{( n g / g ~ d w ) ~}$ \\
\hline AR1 & $24.00 \pm 0.65$ \\
\hline AR3 & $22.47 \pm 0.15$ \\
\hline FR1 & $23.22 \pm 1.23$ \\
\hline FR2 & $15.93 \pm 0.45$ \\
\hline FR5 & $23.12 \pm 0.56$ \\
\hline
\end{tabular}

Data are presented as mean \pm standard deviation

Significant differences between rivers were found for the percentage of $\mathrm{Zr}(p=0.0060)$, $\mathrm{Sr}(p=0.0050)$, Th $(p=0.0084), \mathrm{Pb}(p=0.0352), \mathrm{Zn}(p=0.0263), \mathrm{Ti}(p=0.0446)$ and $\mathrm{Ba}(p=0.0266)$, with the FR reaching higher percentages of these elements (Table 4 ). Percentages of elements $\mathrm{Se}, \mathrm{Hg}, \mathrm{Au}, \mathrm{Co}, \mathrm{Sc}, \mathrm{Cs}, \mathrm{Te}, \mathrm{Cd}, \mathrm{Ag}, \mathrm{Pd}, \mathrm{S}$ and $\mathrm{Sb}$ were below the limit of detection at all sites of both rivers.

Table 4 Percentage of trace elements (Mo, molybdenum; $\mathrm{Zr}$, zirconium; Sr, strontium; U, uranium; Rb, rubidium; Th, thorium; Pb, lead; $\mathrm{Zn}$, zinc; W, tungsten; $\mathrm{Cu}$, cooper; Ni, nickel; Fe, iron; Mn, manganese; $\mathrm{Cr}$, chromium; V, vanadium; Ti, titanium; Ca, calcium; K, potassium; Ba, barium; Sn, tin) present in the sediment of the sampling sites of the Âncora (AR1 to AR5) and Ferreira (FR1 to FR6) rivers, in spring

\begin{tabular}{|c|c|c|c|c|c|c|c|c|c|c|c|c|c|c|c|c|c|c|c|c|}
\hline ites & Mo (\%) & $\operatorname{Zr}(\%)$ & (\%) & (\%) & Rb (\%) & Th (\%) & $\mathbf{P b}(\%)$ & $\mathrm{Zn}(\%)$ & W (\%) & (\%) & $\mathrm{Ni}(\%)$ & $\mathrm{Fe}(\%)$ & $\mathrm{Mn}(\%)$ & $\mathrm{Cr}(\%)$ & V (\%) & (\%) & Ca (\%) & K (\%) & Ba (\%) & Sn (\%) \\
\hline & & & & & & & & & & & & & & & & & & & & \\
\hline AR2 & & & & & & & & & & & & & & & & & & 52 & & . \\
\hline AR3 & & & & 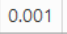 & & & 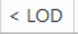 & . & . & & & & & & 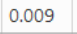 & 6 & & 25 & & 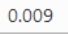 \\
\hline AR4 & 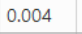 & & 04 & 01 & c & .001 & 0 & 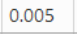 & 0.002 & 005 & & 2.218 & . & & 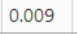 & 0.277 & 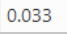 & 76 & . $0<2$ & 0 \\
\hline & & & & & & & & & & & & & & & & & & 08 & & 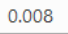 \\
\hline & & & & & & & & & & & & & & & LOD & & & 1 & & .002 \\
\hline & 0.004 & & & & & & & & & & & & & & DD & & & 47 & & \\
\hline FR3 & 0.003 & 0.034 & 0.008 & 2 & 0 & 0.003 & 0.001 & 0.007 & 0.002 & 0.003 & 0.027 & 1.760 & וד & 0.177 & 0.005 & 2 & 0.106 & 69 & 0.022 & 0.002 \\
\hline 104 & 00 & 0 & 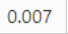 & 002 & 0.018 & 0 & . & 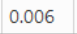 & 0 & 0 & 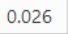 & 政 & 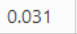 & . & .006 & .300 & t. & 838 & 0220 & 002 \\
\hline S & 0.003 & $0.0<1$ & 0.008 & 01 & c & 0.001 & 0.006 & 0.000 & 0.004 & 0.004 & 0.025 & 3.303 & 0 & 4 & 008 & 91 & .001 & 132 & .029 & .002 \\
\hline FR6 & 004 & JSS & 07 & 001 & 0.017 & 03 & (2) & & 0.004 & 005 & 0.029 & 235 & 041 & 0.219 & 0.009 & 0.322 & 0.041 & 2.784 & 0.022 & 0.002 \\
\hline
\end{tabular}

Percentages of some trace elements determined (S, sulphur; Hg, mercury; Au, gold; Co, cobalt; Sc, scandium; Cs, cesium; Te, tellurium; Cd, cadmium; Ag, silver; Pd, palladium; Sb, antimony; As, arsenic) are not shown because they were below the limit of detection ( $<$ LOD) at all sites of both rivers LOD limit of detection 


\section{Biomarker responses}

Significant seasonal (Table $\underline{8}$ in Appendix 4) and spatial differences were found within each river (identified by stars in Figs. $\underline{2}, \underline{3}$ and $\underline{4}$; Table $\underline{9}$ in Appendix 4) for some of the analysed taxa.

Fig. 2

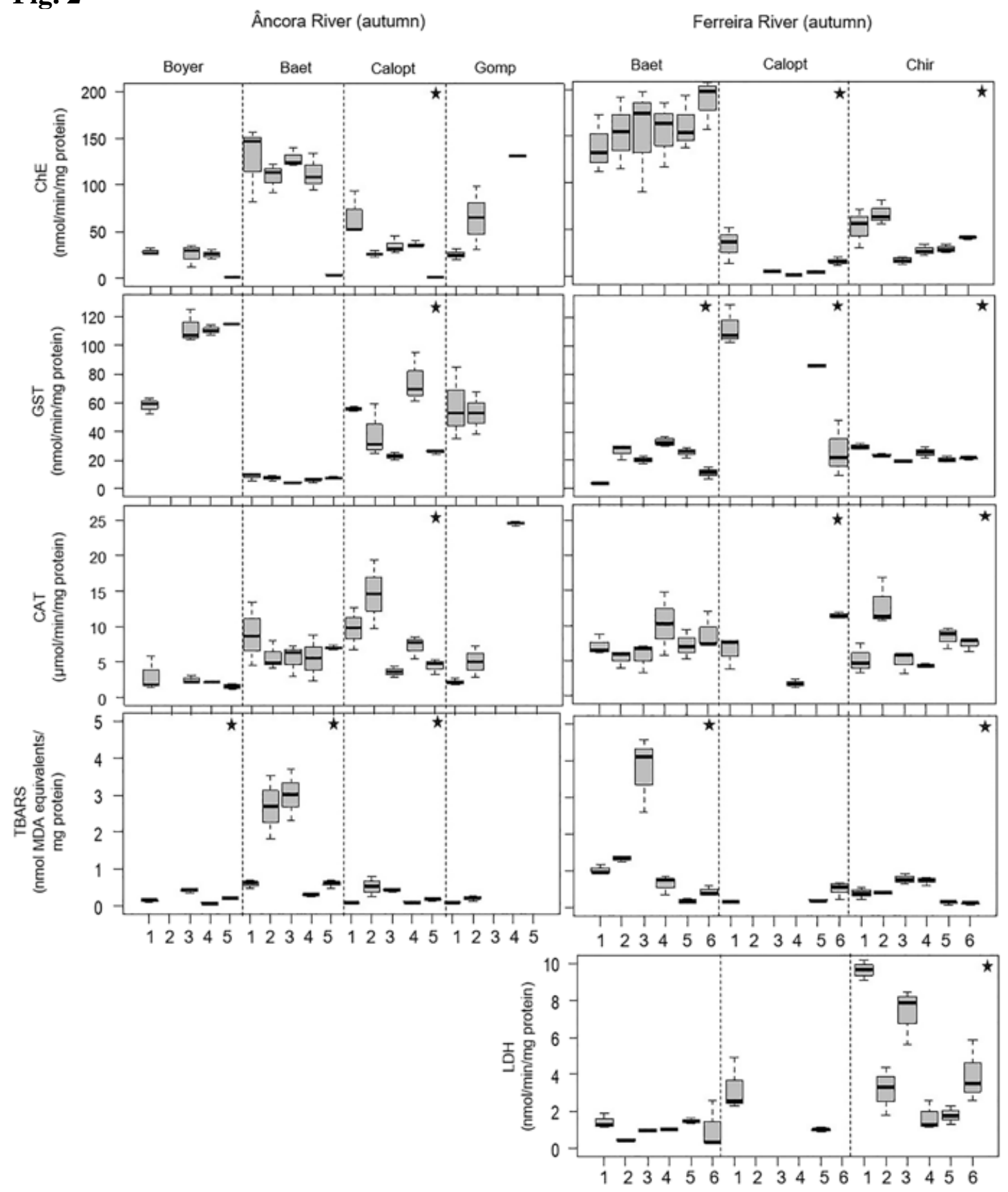

Boxplots of the enzyme activities (cholinesterases, ChE; glutathione $S$-transferases, GST; catalase, CAT; thiobarbituric acid-reactive substances, TBARS; lactate dehydrogenase, LDH) and lipid peroxidation levels (measured as thiobarbituric acid reactive substances, TBARS) in different macroinvertebrate taxa (Baetis spp., Baet; Boyeria spp., Boyer; Caenis spp., Caen; Calopteryx spp., Calopt; Chironomidae, Chir; Gomphus spp., Gomp) sampled from the Âncora and the Ferreira River sites (AR1 to AR5; FR1 to FR6) in autumn. The length of each box shows the range of the central $50 \%$ of the values, with 
the box edges at the first and third quartiles. A star identifies taxa for which biomarker activities differed significantly between sites $(p<0.05)$, according to the non-parametric Kruskal-Wallis test

Fig. 3

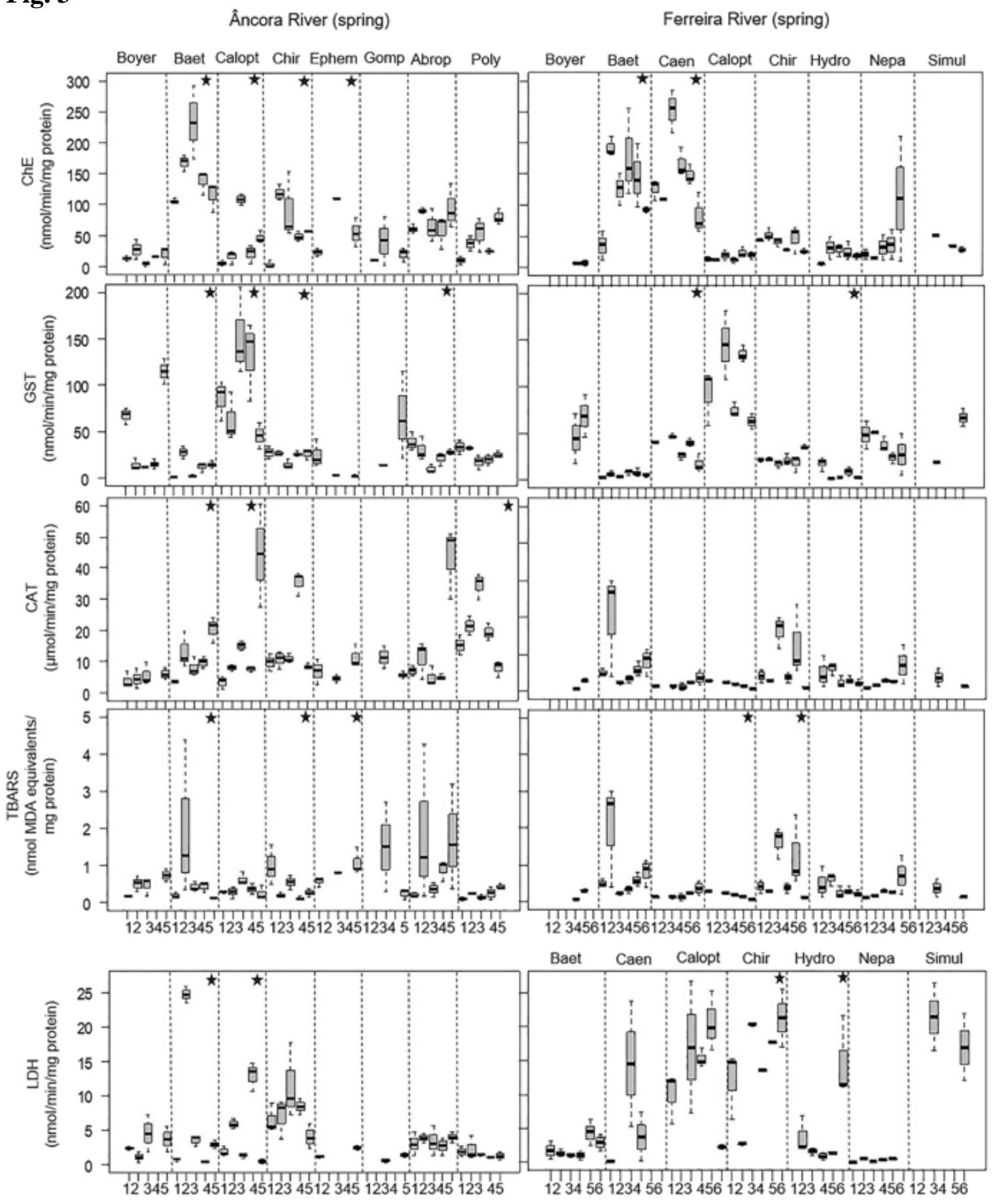

Boxplots of the enzyme activities (cholinesterases, ChE; glutathione $S$-transferases, GST; catalase, CAT; thiobarbituric acid-reactive substances, TBARS; lactate dehydrogenase, $\mathrm{LDH}$ ) and lipid peroxidation levels (measured as thiobarbituric acid reactive substances, 
TBARS) in different macroinvertebrate taxa (Abrophlebia spp., Abrop; Baetis spp., Baet; Boyeria spp., Boyer; Caenis spp., Caen; Calopteryx spp., Calopt; Chironomidae, Chir; Ephemerella spp., Ephem; Gomphus spp., Gomp; Hydropsyche spp., Hydro; Nepa spp., Nepa; Polycentropodidae spp., Poly; Simuliidae, Simul) sampled from the Âncora and the Ferreira River sites (AR1 to AR5; FR1 to FR6) in spring. The length of each box shows the range of the central $50 \%$ of the values, with the box edges at the first and third quartiles. A star identifies taxa for which biomarker activities differed significantly between sites $(p<0.05)$, according to the non-parametric Kruskal-Wallis test

Fig. 4

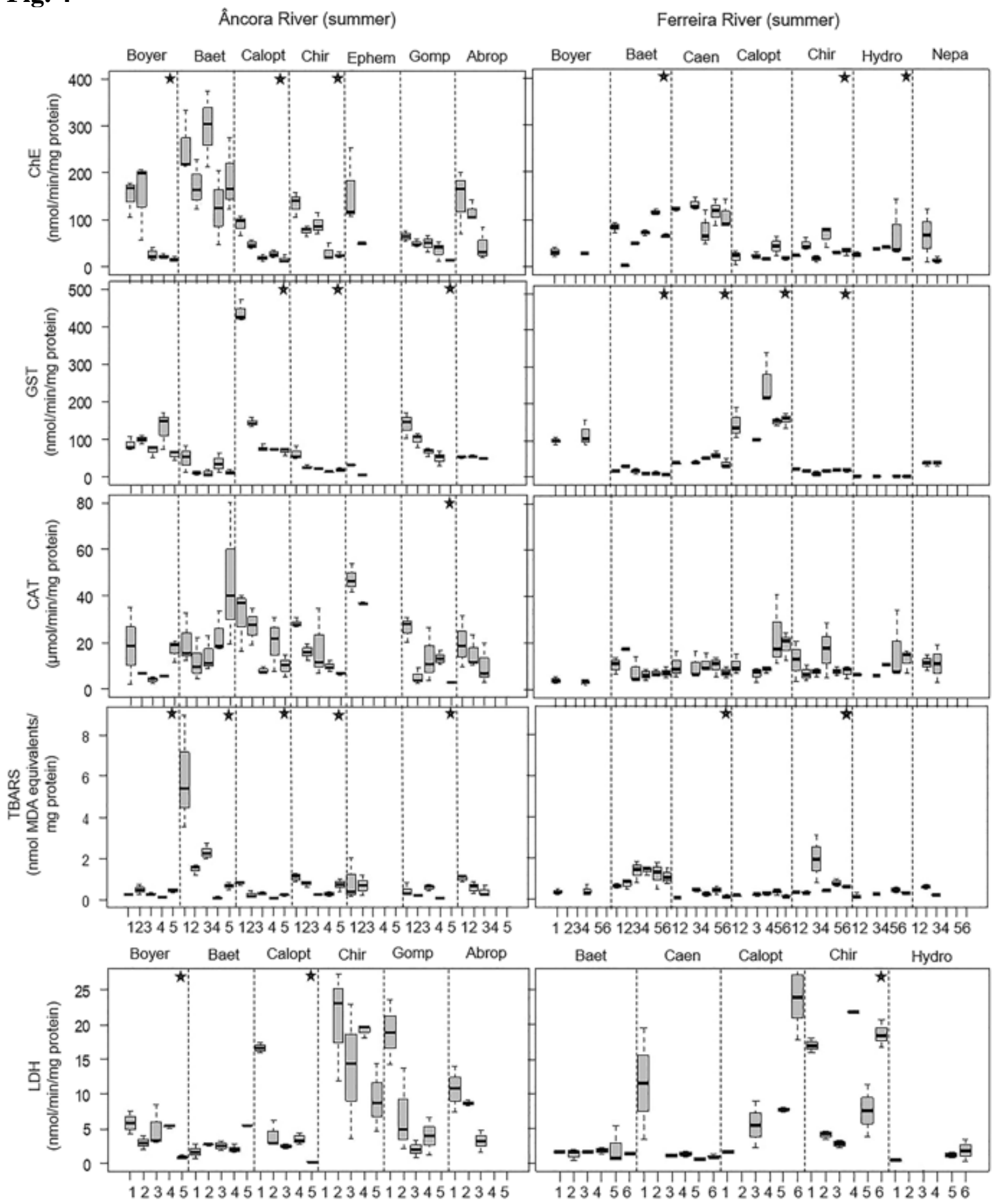


Boxplots of the enzyme activities (cholinesterases, ChE; glutathione $S$-transferases, GST; catalase, CAT; thiobarbituric acid-reactive substances, TBARS; lactate dehydrogenase, $\mathrm{LDH}$ ) and lipid peroxidation levels (measured as thiobarbituric acid reactive substances, TBARS) in different macroinvertebrate taxa (Abrophlebia spp., Abrop; Baetis spp., Baet; Boyeria spp., Boyer; Caenis spp., Caen; Calopteryx spp., Calopt; Chironomidae, Chir; Ephemerella spp., Ephem; Gomphus spp., Gomp; Hydropsyche spp., Hydro; Nepa spp., Nepa) sampled from the Âncora and the Ferreira River sites (AR1 to AR5; FR1 to FR6) in summer. The length of each box shows the range of the central $50 \%$ of the values, with the box edges at the first and third quartiles. A star identifies taxa for which biomarker activities differed significantly between sites $(p<0.05)$, according to the non-parametric Kruskal-Wallis test

In the AR, the medians of ChE activity were, in general, higher for Baetis spp. (103.6$304.3 \mathrm{nmol} / \mathrm{min} / \mathrm{mg}$ protein, except at AR5 in autumn, with a median of $3.5 \mathrm{nmol} / \mathrm{min} / \mathrm{mg}$ protein), compared to the other analysed taxa (Figs. $\underline{2}, \underline{3}$ and $\underline{4}$ ). Most AR1 taxa presented lower medians of ChE activity in spring and higher medians in summer, compared to the other sampling sites (Figs. $\underline{3}$ and $\underline{4}$ ). In addition, significant seasonal differences in $\mathrm{ChE}$ activities ( $p<0.05$; Table $\underline{8}$ in Appendix 4) were found for Boyeria spp. and Baetis spp., with higher activities in summer compared to the spring for Boyeria spp. and compared to autumn and spring for Baetis spp. In the FR, Baetis spp. and Caenis spp. showed higher median values of ChE activity (Baetis spp., 36.0-185.6 nmol/min/mg protein; Caenis spp., $70.8-255.3 \mathrm{nmol} / \mathrm{min} / \mathrm{mg}$ protein) than the remaining taxa (Figs. $\underline{2}, \underline{3}$ and $\underline{4}$ ). In this river, Boyeria spp. and Calopteryx spp. had higher ChE activity in summer compared to the other seasons, and Baetis spp. had higher ChE activity in autumn in comparison to spring and summer $(p<0.05)$.

In the autumn, the medians of GST activity at AR sites were clearly lower for Baetis spp. (4.5-6.6 $\mathrm{nmol} / \mathrm{min} / \mathrm{mg}$ protein) compared to the other taxa analysed (26.6-107.3 nmol/min/mg protein; Fig. 2). In spring, most taxa presented medians of GST activity below $30 \mathrm{nmol} / \mathrm{min} / \mathrm{mg}$ protein, but medians above this value were observed, mainly for Calopteryx spp. (AR1 to AR5, 46.6-135.6 nmol/min/mg protein; Fig. $\underline{3}$ ). In summer, Odonata (Calopteryx spp., Boyeria spp., Gomphus spp. and Abrophlebia spp.) showed medians of GST activity above $40 \mathrm{nmol} / \mathrm{min} / \mathrm{mg}$ protein at almost all sites, especially at AR1 (Fig. 4). Significant seasonal differences in GST activity $(p<0.05$; Table $\underline{8}$ in Appendix 4) in macroinvertebrates from the AR where found for Boyeria spp., with higher activities occurring in autumn compared to spring and summer, for Baetis spp. and Calopteryx spp., with higher values in spring and/or summer compared to autumn, and for Abrophlebia spp., with higher values occurring in summer compared to spring. Higher medians of GST activity were observed in Calopteryx spp. in the FR in all seasons and sites (63.1-151.8 nmol/min/mg protein, except in FR6 in autumn, with a median of 21.9 $\mathrm{nmol} / \mathrm{min} / \mathrm{mg}$ protein), compared to the other analysed taxa (Figs. $\underline{2}, \underline{3}$ and $\underline{4}$ ). In this river, significant differences $(p<0.05)$ in GST activity were found between summer and spring for Boyeria spp. and Caenis spp., with higher values occurring in summer. There were also significant differences $(p<0.05)$ in GST activity between spring and the remaining seasons in Baetis spp., with lower values occurring in spring, and between summer and the remaining seasons in Chironomidae, with lower values occurring in summer. 
The medians of CAT activity in the different taxa analysed in both rivers were mostly below $20 \mu \mathrm{mol} / \mathrm{min} / \mathrm{mg}$ protein, although medians above this value were observed for some taxa in spring and summer, in both rivers (Figs. $\underline{2}, \underline{3}$ and $\underline{4}$ ). For example, almost all taxa showed a median of CAT activity close to or higher than $20 \mu \mathrm{mol} / \mathrm{min} / \mathrm{mg}$ protein at AR1 site in summer $(18.5-46.7 \mu \mathrm{mol} / \mathrm{min} / \mathrm{mg}$ protein, except Baetis spp. with a median of $15.7 \mu \mathrm{mol} / \mathrm{min} / \mathrm{mg}$ protein; Fig. 4). Significant seasonal differences in the activity of this enzyme ( $p<0.05$; Table $\underline{8}$ in Appendix 4) were found in Boyeria spp., Baetis spp., Calopteryx spp. and Ephemerella spp. in the AR, and in Boyeria spp., Baetis spp. and Caenis spp. in the FR, with higher CAT activities occurring in the summer in AR taxa and in the spring in FR taxa.

In both rivers studied, medians of TBARS levels were generally below $2 \mathrm{nmol} / \mathrm{mg}$ protein for all taxa, but higher medians were observed for Baetis spp. in both rivers (e.g. at AR1 in the summer and at FR3 in the autumn with a median of TBARS levels of 5.4 and 4.1 $\mathrm{nmol} / \mathrm{mg}$ protein, respectively; Figs. $\underline{2}, \underline{3}$ and $\underline{4}$ ). TBARS levels only varied significantly among seasons ( $p<0.05$; Table $\underline{8}$ in Appendix 4) for Baetis spp., namely between spring and the remaining seasons in the AR, and between the spring and summer in the FR, with lower levels occurring in the spring in both rivers.

In most AR taxa, medians of LDH activity were, in general, below $10 \mathrm{nmol} / \mathrm{min} / \mathrm{mg}$ protein in the spring $(0.5-9.6 \mathrm{nmol} / \mathrm{min} / \mathrm{mg}$ protein, except in Baetis spp. at AR2 and in Calopteryx spp. at AR4, with medians of $24.7 \mathrm{nmol} / \mathrm{min} / \mathrm{mg}$ protein and 13.6 $\mathrm{nmol} / \mathrm{min} / \mathrm{mg}$ protein, respectively; Fig. 3 ). In summer, the Chironomidae family showed medians close to or higher than $10 \mathrm{nmol} / \mathrm{min} / \mathrm{mg}$ protein at all sites (8.8-26.7 $\mathrm{nmol} / \mathrm{min} / \mathrm{mg}$ protein). In this season, at AR1, most taxa presented activities above 10 $\mathrm{nmol} / \mathrm{min} / \mathrm{mg}$ protein (four of six taxa had medians of LDH activity ranging from 10.8 to $26.7 \mathrm{nmol} / \mathrm{min} / \mathrm{mg}$ protein) (Fig. 4). In the AR, significant seasonal differences $(p<0.05$; Table 8 in Appendix 4) in LDH activity were found for Chironomidae, Gomphus spp. and Abrophlebia spp., with higher values occurring in summer compared to spring. For the FR's taxa, medians of LDH activity were lower than $10 \mathrm{nmol} / \mathrm{min} / \mathrm{mg}$ protein in autumn (Fig. 2). In spring and summer (mainly spring), medians above $10 \mathrm{nmol} / \mathrm{min} / \mathrm{mg}$ protein (11.4-31.8 nmol/min/mg protein) were observed for Chironomidae, Calopteryx spp. and Simuliidae, at most sampling sites (Figs. $\underline{3}$ and $\underline{4}$ ). In this river, significant seasonal differences ( $p<0.05$; Table $\underline{8}$ in Appendix 4$)$ in LDH activity were found for Chironomidae, being this activity lower in autumn compared to the remaining seasons, as well as for Calopteryx spp. and Hydropsyche spp., with higher values occurring in spring compared to autumn for Calopteryx spp. and compared to summer for Hydropsyche spp.

Overall, regarding the biomarker activities in taxa common to both rivers (Chironomidae, Baetis spp., Calopteryx spp. and Boyeria spp.), significant differences between rivers were found for TBARS levels and LDH activity in Calopteryx spp. in spring (TBARS: $p$ $=0.0211$; LDH: $p=0.0472)$; Calopteryx spp. from the AR showed higher levels of TBARS than those from the FR, and Calopteryx spp. from the FR exhibited higher LDH activities than those from the AR. For Baetis spp., cholinesterases (ChE) activities differed significantly between rivers, in both autumn and summer (autumn: $p=0.0062$; summer: $p=0.0062$ ). Higher activities were measured in Baetis spp. from the FR in autumn and in Baetis spp. from the AR in the summer. There were also significant differences between rivers in CAT activity for Baetis spp. in the summer $(p=0.0090)$, with Baetis spp. from the AR showing higher activities than Baetis spp. from the FR. 


\section{Multivariate analysis}

The results of the PCA done to characterise the overall response patterns of Calopteryx spp., Baetis spp. and Chironomidae are presented in Fig. ‥ The first two principle components (PC1 and PC2) extracted summarised 50.3\% of the total variability observed in the data (Fig. 4). Biological variables GST and LDH, CAT and $\mathrm{IPtI}_{N}$, and $\mathrm{ChE}$ and LPO were found to be highly correlated one to the other, respectively. PC1 established a gradient of response of the taxa examined, opposing Calopteryx spp. to Baetis spp. (Fig. 5). This component was linked to biomarkers $\mathrm{ChE}$ and LPO and to the macroinvertebrate index $\left(\operatorname{IPtI}_{\mathrm{N}}\right)$. ChE exhibited a stronger positive correlation with this dimension $(r=0.86$, $p<0.001)$ than LPO $(r=0.67, p<0.001)$ and $\operatorname{IPtI}_{\mathrm{N}}(r=0.47, p<0.001)$. Calopteryx spp. appeared to show lower levels of $\mathrm{ChE}$ and LPO; opposite trends were exhibited by Baetis spp. The results also indicated that taxon responses were stable across seasons (Fig. $\underline{5}$ ). PC2 was linked to GST $(r=0.67, p<0.001)$, to CAT $(r=0.61 p<0.001)$ and, to a lesser extent, to LDH $(r=0.54, p<0.001)$ and $\operatorname{IPtI}_{N}(r=0.44, p<0.001)$, all positively correlated with this dimension. Calopteryx spp. tended to show higher levels of GST, LDH and CAT activities, opposed to the response patterns of Baetis spp. CAT activity tended to be associated with higher scores of the macroinvertebrate index. Overall, the three taxa showed distinct patterns of biological responses, prompting the subsequent analysis of each taxon separately.

\section{Fig. 5}



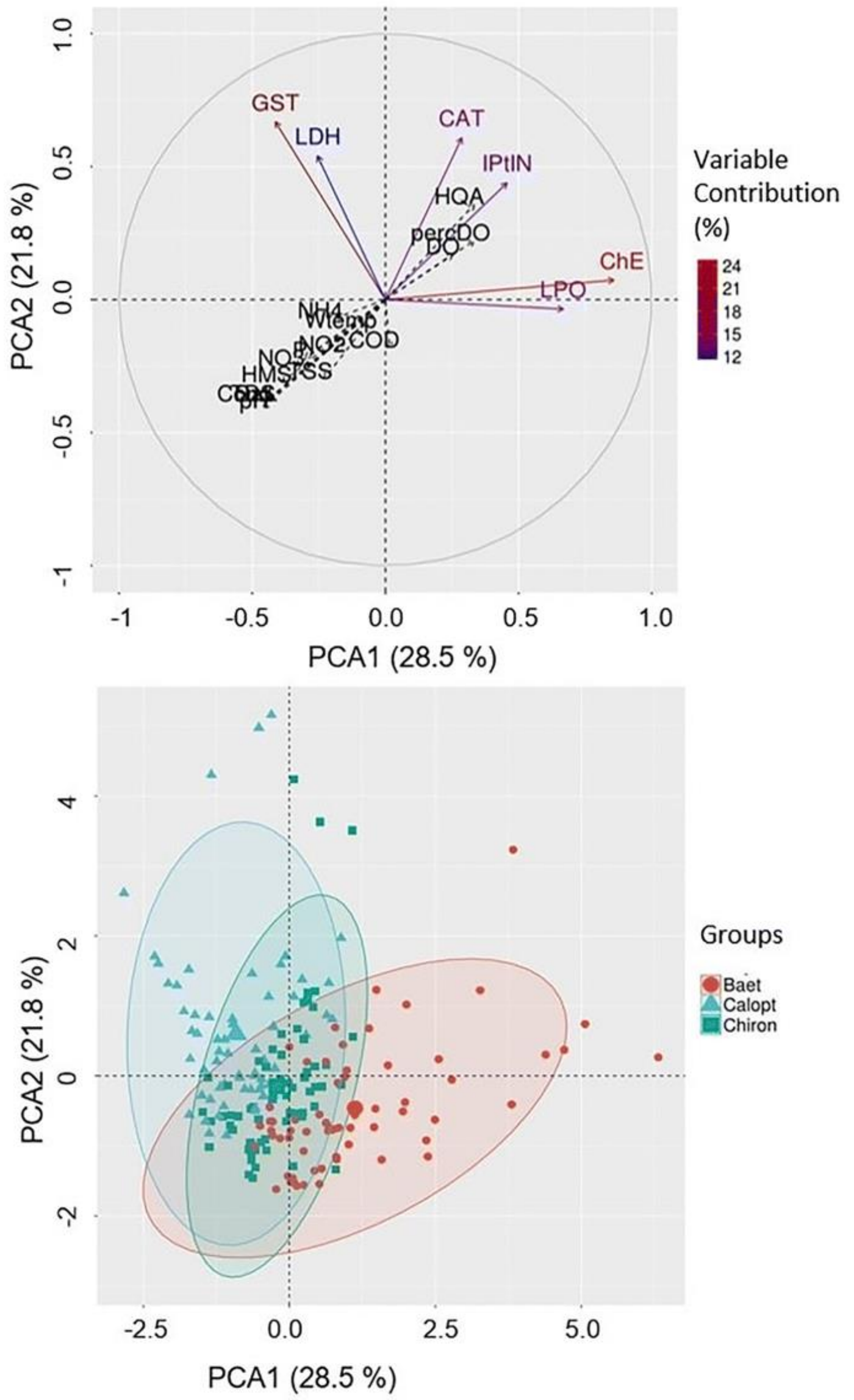
Results of the PCA carried out with taxa and season as qualitative supplementary variables. Qualitative variables were the biomarkers-ChE, GST, CAT, LPO (measured as TBARS levels) and LDH determined in Calopteryx spp., Baetis spp. and Chironomidae - and the North Invertebrate Portuguese Index (IPtI $\left.)_{\mathrm{N}}\right)$. Water physicochemical parameters (water temperature, Temp; $\mathrm{pH}$; dissolved oxygen concentration, DO; percent of saturation of dissolved oxygen, \% DO; chemical oxygen demand, COD; conductivity, Cond; salinity, Sal; total dissolved solids, TDS; total suspended solids, TSS; total phosphorus, $\mathrm{P}$; nitrates, $\mathrm{NO}_{3}{ }^{-}$; nitrites, $\mathrm{NO}_{2}{ }^{-}$; ammonium ion, $\mathrm{NH}_{4}{ }^{+}$) and hydromorphological indices (Habitat Quality Assessment, HQA; Habitat Modification Score, HMS) were included in the analysis as quantitative supplementary variables. Concentration ellipses are shown for each taxon (Baetis spp., Baet; Calopteryx spp., Calopt; Chironomidae, Chiron)

All of the three taxa clearly exhibit distinct response patterns for the two rivers studied (Fig. 6). However, PCA based on either Calopteryx spp. or Chironomidae explained higher percentages of variability in the datasets. Biological responses were also found to be fairly stable across seasons for all of them. Two significant dimensions were extracted for Baetis spp. and Chironomidae, representing $54.2 \%$ and $61.4 \%$ of the overall variability in the dataset, respectively. Three significant dimensions were extracted for Calopteryx spp., expressing $85.2 \%$ of the total variability in the dataset. Overall, for each taxon, PC1 and PC2 summarised a representative amount of the total variance of the dataset (Fig. 6). Hence, interpretation of the results obtained was based on these components. For Calopteryx spp., PC1 established a spatial gradient between the rivers, with LPO, ChE and GST showing strong correlations with this dimension (Fig. $\underline{6}$, top). Although less strongly, IPtI $_{N}$ and CAT were also significantly correlated with PC1. Calopteryx spp. from the FR were under environmental stress, i.e. exposed to higher levels of nitrates, nitrites, ammonia and phosphates and $\mathrm{pH}$. In the FR, this taxon exhibited inhibition of $\mathrm{ChE}$ and CAT activities, unlike in the AR. As previously observed, $\operatorname{IPtI}_{\mathrm{N}}$ values, and consequently the ecological status of this watercourse, were also higher in the AR than in the FR. Conversely, high LPO levels also tended to occur in the AR, suggesting that Calopteryx spp. were under chemical stress. Analysis of the cloud of individuals in the dataset revealed that AR macroinvertebrates showing higher LPO levels were collected at the AR1 and AR2 sampling sites. PC2 further distinguished the two rivers. Biological variables correlated with this dimension were LDH, GST and $\operatorname{IPtI}_{\mathrm{N}}$. Calopteryx spp. from the FR exhibited high LDH and GST activities, particularly those from FR5 and FR6 sites, which presented "poor" ecological status, as indicated by low $\operatorname{IPtI}_{\mathrm{N}}$ index scores (Fig. 6, top). In contrast, for Chironomidae, LDH, ChE and CAT (correlated mainly to PC1) and LPO (correlated to PC2) were the biomarkers producing discrimination of the two rivers (Fig. $\underline{6}$, middle). In the AR, biomarkers associated to PC1 were also associated to higher values of the $\mathrm{IPtI}_{\mathrm{N}}$ index, whereas the FR tended to show inhibition of these biomarkers and higher LPO levels, particularly at some sites.

\section{Fig. 6}


Calopteryx spp.

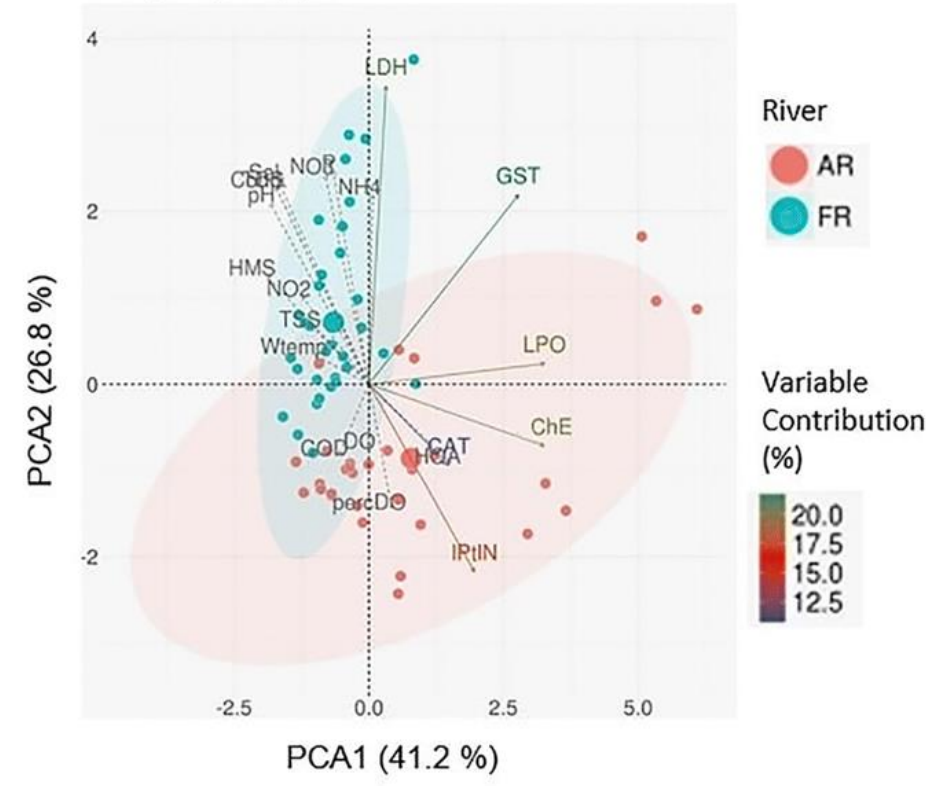

\section{Chironomidae}

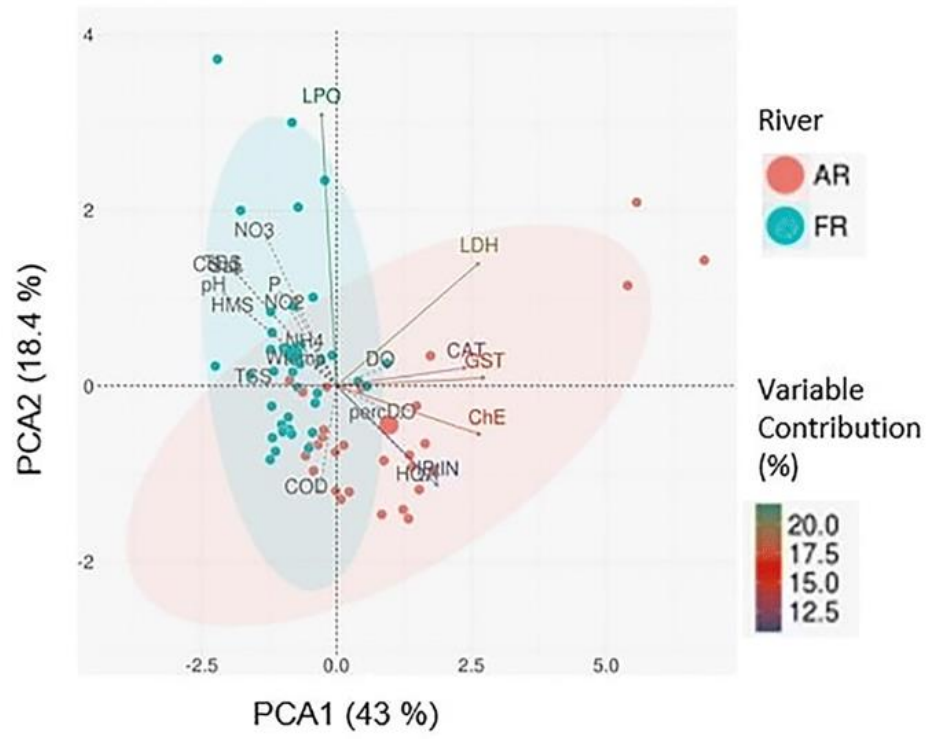

Baetis spp.

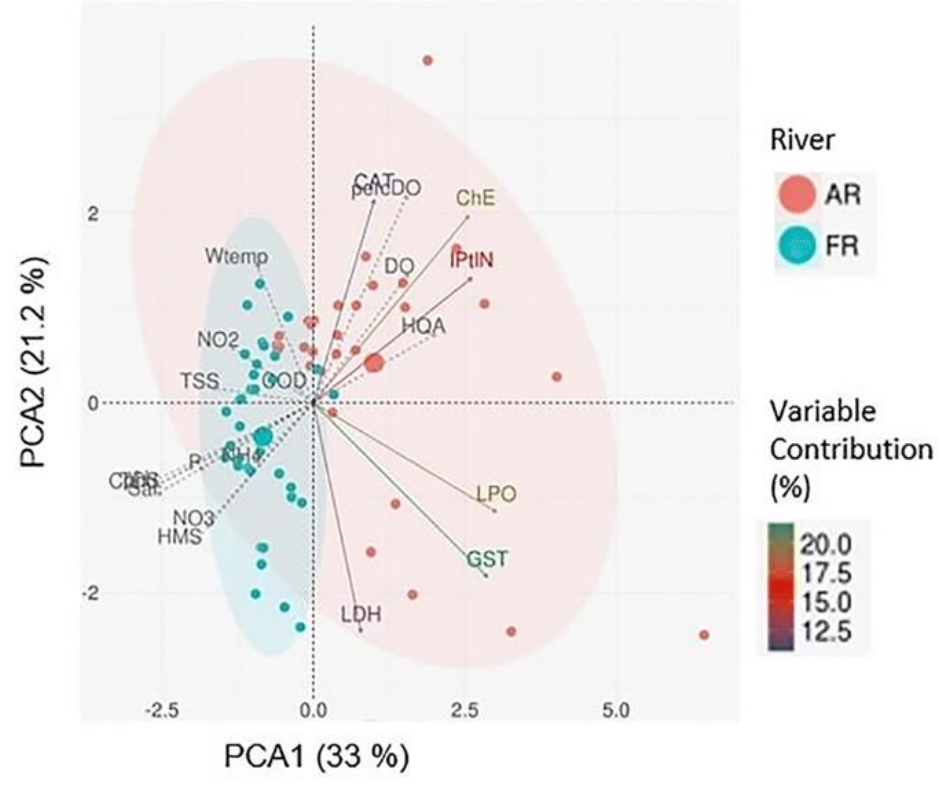


PCA biplots showing the relationships among quantitative biological variables, and the spatial gradients established, for each studied taxon. Concentration ellipses estimated for each river (Âncora River, AR; Ferreira River, FR) are also plotted. Legend as in Fig. $\underline{5}$

\section{Discussion}

Nowadays, the Water Framework Directive (WFD) requires new ecological perspectives with broad multi-disciplinary approaches (Martinez-Haro et al. 2015). In this study, we aimed to understand if a battery of widely recognised biochemical biomarkers, evaluated in different macroinvertebrate groups tolerant and sensitive to pollution, could complement the ecological approaches currently applied in assessing and monitoring the aquatic environment. All the biochemical parameters used in this study could be altered by xenobiotic exposure and were already successfully used as environmental biomarkers in field studies using benthic macroinvertebrates (e.g. Olsen et al. 2001; Berra et al. 2004; Barata et al. 2005; Minutoli et al. 2013; Kaya et al. 2014). Most of these studies employed one single species for biomarker determination. However, since distinct taxonomic groups may have different sensitivities to environmental stressors, the selection of the most adequate bioindicator species/taxon is not straightforward. Hence, this study adopted a multi-taxon and a multi-biomarker evaluation to identify the most informative taxa, together with a consolidated evaluation of the macroinvertebrate community index recommended by the WFD and evaluation of water physico-chemical quality.

\section{Environmental parameters}

Concerning the water physico-chemical parameters, in the AR, levels of phosphorus above the maximum limit value considered "good" for Northern Portuguese rivers (P $>0.10 \mathrm{mg} / \mathrm{L}$; INAG 2009) were observed mainly in spring and summer, which seems to be related to the occurrence of forest fires in the vicinity of some sampling sites (AR1 and AR3), as well as to the increased fertiliser application in agricultural lands close to the river banks (AR2 and AR4). In the FR, the high levels of nutrients (i.e. $\mathrm{NH}_{4}{ }^{+}>1$ $\mathrm{mg} / \mathrm{L} ; \mathrm{NO}_{3}{ }^{-}>25 \mathrm{mg} / \mathrm{L} ; \mathrm{NO}_{2}{ }^{-}>0.1 \mathrm{mg} / \mathrm{L} ; \mathrm{P}>0.10 \mathrm{mg} / \mathrm{L}$; INAG 2009) found in all seasons and sites, particularly at the downstream sites (FR4 to FR6) in spring and summer, seemed to be related not only to agricultural practices but also to discharges from urban areas and wastewater treatment plants.

Organophosphorus pesticides (OPs) are acetylcholinesterase inhibitors and have been widely applied because they are effective insecticides (Girard 2013). Regarding OP analysis in the sediment of the rivers studied, sediment grain size proved to be an important parameter for the extraction and quantification of the pesticides studied (higher recoveries were obtained with grain sizes $\leq 0.25 \mathrm{~mm}$ compared to the grain sizes $\leq 2 \mathrm{~mm}$ ), and only chlorpyrifos was present in $40 \%$ and $50 \%$ of the AR and FR sampling sites, respectively. Chlorpyrifos is extensively used and continuously introduced into the environment being therefore considered a pseudo-persistent organic pollutant (Bonansea et al. 2013; Li et al. 2014). This pesticide has been used as a replacement for other OPs (namely, chlorfenvinphos, diazinon and parathion methyl) banned by the EU (Regulation EC No. 2009/1107) (Terrado et al. 2009). It has been applied over all types of crops (urban and agricultural) and as powder for insect control (Masiá et al. 2015). 
Sediments are habitat and shelter for benthic macroinvertebrates, as well as the major repository of contaminants (organic and inorganic) that reach surface waters (Bettinetti et al. 2012). The Directive 2013/39/EU (EU 2013), which regards priority substances (PS) in the field of water policy, establishes 45 PSs and sets environmental quality standards (EQS) for these pollutants, including chlorpyrifos (with a maximum allowable concentration of $0.1 \mu \mathrm{g} / \mathrm{L}$ ). This directive has also biota EQS for some hydrophobic PS, and for these PS, biota becomes the "default" monitoring matrix (EU 2013). Biota EQS are related mainly to fish for most PS, but for a few PS, biota EQS refer to crustaceans and molluscs or to all three groups. The promotion of the "default" use of a suitable biota matrix as another option to water gives cost-effectiveness and flexibility for the laboratories. It can also counterbalance the analytical challenges of nearly non-detectable levels of several PS in water, as a result of their hydrophobic nature (Dosis et al. 2017). However, no directive has included EQS for pesticides in sediments (as well as soil or sludge) (Kvičalová et al. 2012), even though the need to do it is recognised by the EU (EU 2013).

OPs ought to be monitored because they may elicit damage to benthic organisms (Montuori et al. 2016). Only a few studies in Europe have determined the presence of currently used pesticides in other environmental compartments besides water (e.g. Masiá et al. 2013, 2015; Montuori et al. 2015; Ccanccapa et al. 2016; Cembranel et al. 2017). Regarding the results of those studies, chlorpyrifos is a frequently detected pesticide in the sediments of European rivers, at lower (e.g. Cembranel et al. 2017; Masiá et al. 2013; Montuori et al. 2015) and higher (e.g. Ccanccapa et al., 2016; Masiá et al. 2015) concentrations than those observed in the AR and FR (mean value of the samples that presented chlorpyrifos, $23.2 \mathrm{ng} / \mathrm{g} \mathrm{dw}$ in the AR and $20.8 \mathrm{ng} / \mathrm{g} \mathrm{dw}$ in the FR). For example, Masiá et al. (2015) reported that chlorpyrifos was present in $7 \%$ and $93 \%$ of the samples collected from the Llobregat River (Spain) in September/October 2010 and October/November 2011, respectively, with mean concentrations of $0.39 \mathrm{ng} / \mathrm{g} \mathrm{dw}$ and $26.13 \mathrm{ng} / \mathrm{g} \mathrm{dw}$, respectively (concentrations up to $131 \mathrm{ng} / \mathrm{g} \mathrm{dw}$ ). Ccanccapa et al. (2016) also found this pesticide in all sediment samples collected from the Júcar River (Spain) in September/October 2010 and October/November 2011, with median concentrations of $2 \mathrm{ng} / \mathrm{g} \mathrm{dw}$ and $3.15 \mathrm{ng} / \mathrm{g} \mathrm{dw}$, respectively, and in $22 \%$ and $11 \%$ of the sediment samples collected from the Tugar River in September/October 2012 and October/November 2013, with median concentrations of $63.75 \mathrm{ng} / \mathrm{g} \mathrm{dw}$ and below $0.01 \mathrm{ng} / \mathrm{g} \mathrm{dw}$, respectively (Ccanccapa et al. 2016).

Here, the analysis of pesticides in the sediments of both rivers was carried out based on a single campaign in spring. However, long-term data is essential to assess global changes in fluvial systems, since the transport of pesticides to environmental compartments such as sediments is affected by aspects that change over time such as hydrological (e.g. flow, depth) and climatic (e.g. precipitation, wind, temperature) conditions and closeness of agricultural lands to surface waters (Ccanccapa et al. 2016).

Several of the elements that were present in sediments reflect the industrial and other activities in surrounding areas. Some fertilisers and pesticides used in agriculture near the river banks of both rivers studied contain elements such as $\mathrm{K}, \mathrm{Fe}, \mathrm{S}, \mathrm{Ca}, \mathrm{Mg}$ and $\mathrm{Cu}$. Higher percentages of some heavy metals, namely $\mathrm{Zr}$, Th, $\mathrm{Pb}, \mathrm{Zn}, \mathrm{Ti}$ and $\mathrm{Ba}$, were found in the FR compared to the AR, as a result of higher anthropic pressures in the FR (e.g. higher industrial activity). Sediments of both rivers were mainly composed by coarser particles (pebble and granule, very coarse and coarse sand, $>80 \%$ in the AR and $>60 \%$ 
in the FR; Rodrigues et al., submitted) to which trace metals have low affinity, thus contributing to the low retention of trace elements in sediments (Eggleton and Thomas 2004). Although the percentage of heavy metals in the sediment of both rivers was low or not detected, a quantitative analysis of trace elements (e.g. using the analytical technique ICP-MS) is recommendable, since it is much more sensitive than the qualitative analysis used is this study, and heavy metals (most of the trace elements analysed) are highly persistent and can be toxic to life even in trace amounts.

\section{Biological parameters}

Although FR sites showed lower ecological status than AR sites (Rodrigues et al., submitted), not all taxa common to both rivers showed significant differences between rivers regarding biomarker responses. Baetis spp. and Calopteryx spp. showed significant differences between rivers regarding some biomarker activities/levels, although not in all seasons, while Chironomidae and Boyeria spp. showed similar biomarker responses in both rivers, for all seasons.

Overall, the here-evaluated biomarker responses observed in macroinvertebrate taxa showed generally low levels of variation, though they differed between rivers. They also were of lower magnitude compared to those observed in other studies in which the same biomarkers were measured in macroinvertebrates sampled from polluted Mediterranean rivers (Barata et al. 2005; Puertolas et al. 2010; Damásio et al. 2011; Prat et al. 2013). $\mathrm{ChE}$ and CAT activities found in this study were within the ranges reported by Berra et al. (2004), who performed the first attempt to evaluate the basal-level activities of ChE, CAT and GST in different macroinvertebrate families collected from two Italian rivers. However, the GST activities observed in this study were, in general, of lower magnitude than those reported by Berra et al. (2004). It is well known, though, that species and/or populations of distinct genetic make-up, geographic regions and/or previous history of exposure to environmental contamination may exhibit differences in biochemical and physiological parameters (Boets et al. 2012; Jin et al. 2012). This further highlights the importance of characterising local baseline responses and the need for site-specific evaluations.

Results of the present study showed that specific biomarker levels varied from taxon to taxon. This has also been previously observed in other works (Berra et al. 2004; Bonzini et al. 2008), as species' response to pollutants differ depending on their biotransformation capacity, feeding, type of habitat, trophic level and other environmental factors (Ramos et al. 2015). For example, organisms of two genera of the Ephemeroptera order, thought to be in the mid-range for tolerance to many environmental stressors (Harrington and Born 2000; Menetrey et al. 2008), namely Baetis spp. (analysed for both rivers) and Caenis spp. (analysed only for the FR), showed higher ChE activities compared to the other taxa analysed. The differential sensitivity of $\mathrm{ChE}$ found for various taxa suggests they may respond with different intensities when exposed to toxicants able to interfere with the enzyme activity, probably due to differential inhibition constants (Berra et al. 2004). Another example is that organisms of the Odonata order analysed in both rivers (Calopteryx spp., Boyeria spp., Gomphus spp. and Abrophlebia spp.), which are considered sensitive to organic pollution (Alba-Tercedor and Sánchez-Ortega 1988), displayed higher GST activities (especially Calopteryx spp. in the FR) in all seasons compared to the other taxa analysed. Higher GST activities in Odonata compared to other benthic macroinvertebrate taxonomic groups (e.g. Ephemeroptera, Diptera, Plecoptera 
and Trichoptera) were also observed by Berra et al. (2004), and this seems to indicate that Odonata have greater biotransformation capabilities, compared to other taxa.

In both rivers studied, significant spatial differences in biomarker responses within the same season were observed for some taxa. Nevertheless, a single analysis of the responses observed was not able to differentiate sites with different ecological status, as happened in other studies (e.g. Damásio et al. 2011). There were, however, situations in which biomarkers detected biological sub-lethal effects of chemical or other stress exposure (i.e. effects that are not detected at the community level), rather than those associated with high nutrient concentrations and habitat degradation, thus complementing the information given by the ecological quality monitoring procedures currently used in the scope of the WFD. In autumn, AR2 and AR5 sites had "moderate" ecological status, according to the IPtI $_{N}$ index, while the remaining sites had "good" ecological status (Rodrigues et al., submitted). At AR5 in autumn, all macroinvertebrate taxa analysed (including Baetis spp.) showed decreased ChE activities compared to the other AR sites, which seemed to be associated with pesticide inputs resulting from ground leaching caused by rainfall (agricultural land near the river banks) or to interactions of contaminants and environmental factors. Apparently, this contamination did not persist in spring and summer, pointing out that the contamination sources which caused the observed biochemical changes were not acting continuously. None of the studied OPs was found at AR5 in spring. In this season, only AR4 did not show "good" ecological status according to the $\operatorname{IPtI}_{\mathrm{N}}$ index (Rodrigues et al., submitted) but higher inhibition of ChE was observed at AR1 (compared to the other AR sites), where the highest concentration of chlorpyrifos was also detected $(24 \mathrm{ng} / \mathrm{g} \mathrm{dw})$. The pattern of ChE inhibition found in most taxa was not observed at AR3 or in FR sites, where chlorpyrifos was detected at apparently lower concentrations. In fact, the occurrence of a particular contaminant in the environment does not mean that this contaminant is bioavailable, and thus, no conclusion can be drawn regarding its possible harmful biological effects, or even any measurable biological effects (Lam 2009). Therefore, the biomarker approach is useful to complement chemical analysis, providing early-warning information about the exposure to and/or effects of contaminants on organisms and the possible need for more detailed investigations to be carried out. The AR1 site in summer was the only AR site/season with "high" ecological status according to the $\operatorname{IPtI}_{\mathrm{N}}$ index (other sites had "good" ecological status). Here, higher median values of activities of enzymes responsible for the normal neural function (ChE) were found, compared to the remaining sites and seasons, although AR1 also showed higher levels than other sites of biotransformation (GST), antioxidant (CAT) and metabolic (LDH) enzyme activities in most taxa analysed. In this regard, it is noteworthy that several forest fires occurred in a nearby area of AR 1 in the summer. Deposition of atmospheric particulate matter resulting from organic combustion may have triggered the activity of those enzymes to cope with the exposure. Although TBARS levels at all sites were of low magnitude, compared to those observed in macroinvertebrates from polluted rivers and streams (Barata et al. 2005; Puertolas et al. 2010; Damásio et al. 2011; Prat et al. 2013), the highest TBARS levels recorded in this study occurred in Baetis spp. at AR1 in summer.

\section{Multivariate analysis}

Results suggest that employing a battery of well-established biomarkers, measured in different macroinvertebrate taxa with different sensitivities to environmental stressors, gives a more complementary and integrative perception of ecosystem health than the use 
of a single taxon, by encompassing multiple exposure routes, several forms of biological integration of environmental cues and distinct taxa sensitivities. However, for a continuous cost-effective biological monitoring of rivers, the choice of adequate taxa and sampling seasons is relevant. Baetis spp., Chironomidae and especially Calopteryx spp. seemed to be more sensitive in detecting subtle gradients of toxic substances and their effects than the remaining taxa analysed. These organisms were widespread in the studied areas and abundant or bigger sized, providing sufficient biological material for the measurement of the whole battery of biomarkers, in most or all sampling sites and seasons.

The PCA done to investigate overall response patterns of Calopteryx spp., Baetis spp. and Chironomidae showed that biomarker responses in all taxa were stable across seasons (summer and spring) and clearly distinguished Calopteryx spp. from Baetis spp. patterns of biological response to contamination. Calopteryx spp. exhibited lower levels of ChE and LPO and higher levels of GST, LDH and CAT activities, while Baetis spp. showed opposite trends.

The PCAs of the biomarkers measured in Calopteryx spp. and Chironomidae individually clearly discriminated the FR (with low ecological quality) from the AR (higher ecological quality). For example, Calopteryx spp. from the FR exhibited inhibition of ChE and CAT, as opposed to Calopteryx spp. from the AR. This taxon also showed higher GST and LDH activities in the FR compared to AR, particularly at the downstream sites (FR5 and FR6), which presented the worst $\mathrm{IPtI}_{\mathrm{N}}$ scores, as well as higher levels of nutrients. Acetylcholinesterase (AChE) belongs to the cholinesterase family of enzymes (ChEs), which has been detected in both vertebrates and invertebrates. It acts on the hydrolysis of the acetylcholine neurotransmitter at cholinergic synapses and neuromuscular junctions. Inhibition of its activity is known to cause neurotoxic effects. Organophosphorus and carbamate pesticides are specific AChE inhibitors that cause deleterious effects on the target organisms. High inhibition by these anti-cholinesterasic agents leads to death of the exposed animals. However, AChE inhibitors can also be inhibited by other pollutants (such as some metals and PAHs), contaminants of emerging concern (as pharmaceuticals), or even undetermined complex mixtures of contaminants (Payne et al. 1996; Garcia et al. 2000; Schulz and Liess 2000; Berra et al. 2006; Pestana et al. 2009; Domingues et al. 2010; Siebel et al. 2010; Damásio et al. 2011; Santos et al. 2012). CAT is one of the enzymes most responsive to reactive oxygen species (ROS) in both vertebrates and invertebrates (Halliwell and Gutteridge 1999). Various toxicants, such as pesticides, metals (Stohs and Bagghi 1995) and polychlorinated biphenyls (PCBs) (Winston and Di Giulio 1991; Orbea et al. 2002), provoke oxidative stress by increasing the production of ROS (Barata et al. 2005; Sahan et al. 2010). Oxidative damage occurs when the production of ROS and its elimination by the antioxidant defences are unbalanced (Halliwell and Gutteridge 1999; Lushchak 2011). The GST enzymatic complex acts on detoxification of xenobiotics (e.g. PAHs, mixtures of pollutants, pesticides, oils) and is a defence against oxidative damage (Saenz et al. 2010). LDH plays a role in the anaerobic energy production, and its induction is an indication that additional energy is required to readily deal with the chemical stress caused by exposure to contaminants (DeCoen and Janssen 1997; Jo et al. 2001; Rodrigues et al. 2013, 2015). Thus, its association with the previous mentioned enzymes is also reasonable, suggesting that Calopteryx spp. from the FR, especially at FR5 and FR6, may have been exposed to a low concentration of xenobiotic(s) but were able to cope with the exposure by obtaining additional energy for antioxidant protection and detoxification processes, since no 
oxidative damage was observed. Lipid peroxidation, measured in this study as TBARS levels, is a very relevant outcome of oxidative stress, which can lead to changes in the physico-chemical properties of cellular membranes and deterioration of the cell, which, in turn, disrupts vital functions (Rikans and Hornbrook 1997). Higher LPO levels were observed in the AR, particularly at the AR1 and AR3 sites, probably as a consequence of forest fires that occurred upstream of AR1 and near AR3, suggesting that the organisms were under chemical stress. The PCAs also revealed little seasonal variation in the biological variables (both the biomarkers and the macroinvertebrate community index), suggesting that, under the present climate scenario, monitoring in either season may provide sufficient informative data for the weight-of-evidence approach adopted. Future work should hence also focus on further investigating possible seasonal gradients of response occurring in the AR and FR during these and the other seasons of the year. Successive sampling may provide enough samples of Calopteryx spp. or Chironomidae (these taxa allowed to discriminate responses between rivers and provided useful information to identify specific sampling sites under higher environmental stress) for this purpose.

As to the taxonomic resolution for biomarker analysis, though toxic effects on biota are known to be most noticeable at lower levels (Rubal et al. 2009), the identification of benthic macroinvertebrate species is extremely time-consuming and expensive (Marshall et al. 2006). Also, because of morphological immaturity, some individuals cannot be identified to species level; some specimens may also be cryptic or represent little known groups (Cook et al. 2008; Liu et al. 2003; Pfrender et al. 2010; Weiss et al. 2014). A good example is the immature stages of chironomids. These are usually the most speciesdiverse and abundant freshwater macroinvertebrates, but their identification to species or even genus level is technically very difficult or impossible using traditional morphologybased methods (Jones 2008). The present results support the notion that determination of biomarkers in higher-level taxa constitutes an expedite, rapid and cost-effective approach, useful for their integration in monitoring programmes for ecological quality assessment.

Globally, the biological information provided by biomarkers is essential to evaluate toxic effects and conveys the results of the exposure to various stressors, including known and unknown chemicals to which animals are exposed (Guimarães et al. 2011). Finally, biological monitoring of AR and FR sites should be continued, in order to verify how the state of organisms or ecosystem health is progressing and to take timely mitigation actions, if necessary. That would help avoiding that biological effects of contaminants may result in irreversible long-term changes, including biodiversity loss due to the disappearing of sensitive taxa.

\section{Conclusions}

Overall, biomarker responses obtained in both studied rivers indicated that sub-lethal biological effects may occur in apparently healthy ecosystems, such as the AR (e.g. higher LPO levels in Calopteryx spp. and Baetidae spp. at AR1). The results further indicate that the use of a multi-biomarker battery, sensitive to water contamination, may give information to complement the diagnosis of future ecological impairment or to establish reference sites. Results also suggest that a set of well-established biomarkers measured in different macroinvertebrate taxa provides a global complementary view of ecosystem health, as it integrates several exposure routes and forms of biotic integration of the environment, as well as distinct taxa sensitivities. Calopteryx spp., Chironomidae and 
Baetis spp. and the spring and summer were the most useful taxa and seasons for multivariate analysis, which showed distinct patterns of biological response in the three taxa. The integrated analysis indicated that the most useful taxa to implement a costeffective application of biomarkers for diagnostic purposes would be Calopteryx spp. and Chironomidae. These taxa allowed to discriminate responses among rivers and provided useful information to identify specific sampling sites under higher environmental stress. Looking at these taxa, with a wider distribution, abundant and/or of bigger body size, will help minimise problems, such as missing values, high dimensionality and difficulty in obtaining complete data for some taxa (resulting from their high sensitivity to environmental quality). Each of these taxa presented clear biological response patterns for the two rivers studied, which reflected different ecological statuses in line with the macroinvertebrate index recommended by the WFD.

\section{References}

1. Aebi, H. (1984). Catalase in vitro. Methods in Enzymology, 105, 121-126. https://doi.org/10.1016/S0076-6879(84)05016-3.

2. Alba-Tercedor, J., \& Sánchez-Ortega, A. (1988). Un método rápido y simple para evaluar la calidad biológica de las aguas corrientes basado en el de Hellawell (1978). Limnetica, 4, 51-56 (in Spanish).

3. Allan, I. J., Vrana, B., Greenwood, R., Mills, G. A., Roig, B., \& Gonzalez, C. (2006). A "toolbox" for biological and chemical monitoring requirements for the European Union's Water Framework Directive. Talanta, 69, 302-322. https://doi.org/10.1016/j.talanta.2005.09.043.

4. APHA (American Public Health Association). (1992). Standard methods for the examination of water and wastewater. Washington, DC: American Public Health Association.

5. Barata, C., Lekumberri, I., Vila-Escale, M., Prat, N., \& Porte, C. (2005). Trace metal concentration, antioxidant enzyme activities and susceptibility to oxidative stress in the tricoptera larvae Hydropsyche exocellata from the Llobregat river basin (NE Spain). Aquatic Toxicology, 74, 3-19. https://doi.org/10.1016/j.aquatox.2005.04.002.

6. Berra, E., Forcella, M., Giacchini, R., Marziali, L., Rossaro, B., \& Parenti, P. (2004). Evaluation of enzyme biomarkers in freshwater invertebrates from Taro 
and Ticino river, Italy. Annales de Limnologie - International Journal of Limnology, 40, 169-180.

7. Berra, E., Forcella, M., Giacchini, R., Rossaro, B., \& Parenti, P. (2006). Biomarkers in caddisfly larvae of the species Hydropsyche pellucidula (Curtis, 1834) (Trichoptera: Hydropsychidae) measured in natural populations and after short term exposure to fenitrothion. Bulletin of Environmental Contamination and Toxicology, 76, 863-870. https://doi.org/10.1007/s00128-006-0998-7.

8. Bettinetti, R., Ponti, B., Marziali, L., \& Rossaro, B. (2012). Biomonitoring of lake sediments using benthic macroinvertebrates. TrAC-Trends in Analytical Chemistry, 36, 92-102. https://doi.org/10.1016/j.trac.2011.12.008.

9. Boets, P., Lock, K., Goethals, P. L., Janssen, C. R., \& De Schamphelaere, K. A. (2012). A comparison of the short-term toxicity of cadmium to indigenous and alien gammarid species. Ecotoxicology, 21, 1135-1144. https://doi.org/10.1007/s10646-012-0868-5.

10. Bonansea, R. I., Amé, M. V., \& Wunderlin, D. A. (2013). Determination of priority pesticides in water samples combining SPE and SPME coupled to GCMS. A case study: Suquía River basin (Argentina). Chemosphere, 90(6), 18601869. https://doi.org/10.1016/j.chemosphere.2012.10.007.

11. Bonzini, S., Finizio, A., Berra, E., Forcella, M., Parenti, P., \& Vighi, M. (2008). Effects of river pollution on the colonisation of artificial substrates by macrozoobenthos. Aquatic Toxicology, $\quad 89, \quad 1-10$. https://doi.org/10.1016/j.aquatox.2008.05.008.

\section{$\underline{\text { CAS }} \underline{\text { Article Google Scholar }}$}

12. Bradford, M. M. (1976). A rapid and sensitive method for the quantification of microgram quantities of protein utilizing the principle of protein-dye binding. Analytical Biochemistry, 72, 248-254. https://doi.org/10.1016/00032697(76)90527-3.

13. Buege, J. A., \& Aust, S. D. (1978). Microssomal lipid peroxidation. Methods in Enzymology, 52, 302-310. https://doi.org/10.1016/S0076-6879(78)52032-6. 
14. Ccanccapa, A., Masiá, A., Andreu, V., \& Picó, Y. (2016). Spatio-temporal patterns of pesticide residues in the Turia and Jucar rivers (Spain). Science of the Environment, 540 , 200-210. https://doi.org/10.1016/j.scitotenv.2015.06.063.

15. Cembranel, A. S., Sampaio, S. C., Remor, M. B., Gotardo, J. T., \& Rosa, P. M. D. (2017). Geochemical background in an oxisol. Engenharia Agrícola, 37(3), 565-573. https://doi.org/10.1590/1809-4430-eng.agric.v37n3p565-573/2017.

16. Chambers, J. M., Cleveland, W. S., Kleiner, B., \& Tukey, P. A. (1983). Graphical methods for data analysis. Belmont: Wadsworth International Group.

17. Colin, N., Porte, C., Fernandes, D., Barata, C., Padrós, F., Carrassón, M., Monroy, M., Cano-Rocabayera, O., de Sostoa, A., Piña, B., \& Maceda-Veiga, A. (2016). Ecological relevance of biomarkers in monitoring studies of macro-invertebrates and fish in Mediterranean rivers. Science of the Total Environment, 540, 307-323. https://doi.org/10.1016/j.scitotenv.2015.06.099.

18. Cook, B. D., Page, T. J., \& Hughes, J. M. (2008). Importance of cryptic species for identifying 'representative' units of biodiversity for freshwater conservation. Biological Conservation, 141, 2821-2831. https://doi.org/10.1016/j.biocon.2008.08.018.

19. Correia-Sá, L., Fernandes, V. C., Carvalho, M., Calhau, C., Domingues, V. F., \& Delerue-Matos, C. (2012). Optimization of QuEChERS method for the analysis of organochlorine pesticides in soils with diverse organic matter: sample preparation. Journal of Separation Science, 35(12), 1521-1530. https://doi.org/10.1002/jssc.201200087.

20. Damásio, J., Fernandez-Sanjuan, M., Sanchez-Avila, J., Lacorte, S., Prat, N., Rieradevall, M., Soares, A., \& Barata, C. (2011). Multi-biochemical responses of benthic macroinvertebrate species as a complementary tool to diagnose the cause of community impairment in polluted rivers. Water Research, 45, 3599-3613. https://doi.org/10.1016/j.watres.2011.04.006. 
21. De Castro-Catala, N., Munoz, I., Armendariz, L., Campos, B., Barcelo, D., LopezDoval, J., Perez, S., Petrovic, M., Pico, Y., \& Riera, J. L. (2015). Invertebrate community responses to emerging water pollutants in Iberian river basins. Science of the Total Environment, 503, 142-150. https://doi.org/10.1016/j.scitotenv.2014.06.110.

22. DeCoen, W. M., \& Janssen, C. R. (1997). The use of biomarkers in Daphnia magna toxicity testing II. Digestive enzyme activity in Daphnia magna exposed to sublethal concentrations of cadmium, chromium and mercury. Chemosphere, 35, 1053-1067. https://doi.org/10.1016/S0045-6535(97)00172-0.

23. Diamantino, T. C., Almeida, E., Soares, A., \& Guilhermino, L. (2001). Lactate dehydrogenase activity as an effect criterion in toxicity tests with Daphnia magna Straus. Chemosphere, 45, 553-560. $\quad$ https://doi.org/10.1016/S0045$\underline{6535(01) 00029-7 .}$.

24. Domingues, I., Agra, A. R., Monaghan, K., Soares, A., \& Nogueira, A. J. A. (2010). Cholinesterase and glutathione-S-transferase activities in freshwater invertebrates as biomarkers to assess pesticide contamination. Environmental Toxicology and Chemistry, 29, 5-18. https://doi.org/10.1002/etc.23.

25. Dosis, I., Ricci, M., Majoros, L., Lava, R., Emteborg, H., Held, A., \& Emons, H. (2017). Addressing analytical challenges of the environmental monitoring for the Water Framework Directive: ERM-CE100, a new biota certified reference material. Analytical Chemistry, 89(4), 2514-2521. https://doi.org/10.1021/acs.analchem.6b04682.

26. EC (European Commission). (2000). Directive 2000/60/EC of the European Parliament and of the Council of 23rd October 2000 establishing a framework for community action in the field of water policy. Official Journal of the European Communities, L327, 1-73.

27. EC (European Commission). (2013). Commission Decision of 20 September 2013 establishing, pursuant to Directive 2000/60/EC of the European Parliament and of the Council, the values of the Member State monitoring system classifications as a result of the intercalibration exercise and repealing Decision 2008/915/EC. Official Journal of European Union, L266, 1-47. 
28. Eggleton, J., \& Thomas, K. V. (2004). A review of factors affecting the release and bioavailability of contaminants during sediment disturbance events. $\begin{array}{llll}\text { Environment } \quad \text { International, } & \text { 973-980. }\end{array}$ https://doi.org/10.1016/j.envint.2004.03.001.

29. Ellman, G. L., Courtney, K. D., Andres, V., Jr., \& Featherstone, R. M. (1961). A new and rapid colorimetric determination of acetylcholinesterase activity. Biochemical Pharmacology, 7, 88-90. https://doi.org/10.1016/00062952(61)90145-9.

30. EU (European Union). (2013). Directive 2013/39/EU of the European Parliament and of the Council of 12 August 2013 amending Directives 2000/60/EC and 2008/105/EC as regards priority substances in the field of water policy 2013/39/EU. Official Journal of European Union, L226, 1-47.

31. Fernandes, V. C., Domingues, V. F., Mateus, N., \& Delerue-Matos, C. (2013). Multiresidue pesticides analysis in soils using modified QuEChERS with disposable pipette extraction and dispersive solid-phase extraction. Journal of Separation Science, 36, 376-382. https://doi.org/10.1002/jssc.201200673.

32. Fox, J., \& Bouchet-Valat, M. (2018). Rcmdr: R Commander. R package version 2.4-4.

33. Frasco, M. F., \& Guilhermino, L. (2002). Effects of dimethoate and betanaphthoflavone on selected biomarkers of Poecilia reticulata. Fish Physiology and Biochemistry, 26, 149-156. https://doi.org/10.1023/A:1025457831923.

34. Garcia, L. M., Castro, B., \& Guilhermino, L. (2000). Characterization of cholinesterase from guppy (Poecilia reticulata) muscle and its in vitro inhibition by environmental contaminants. Biomarkers, 5, 274-284. https://doi.org/10.1080/135475000413827.

35. Girard, J. (2013). Princípios da química ambiental. Rio de Janeiro: LTC. 
36. Guilhermino, L., Lopes, M. C., Carvalho, A. P., \& Soares, A. M. (1996). Acetylcholinesterase activity in juveniles of Daphnia magna Straus. Bulletin of Environmental Contamination and Toxicology, 57, 979-985. https://doi.org/10.1007/s001289900286.

37. Guimarães, L., Medina, M. H., \& Guilhermino, L. (2011). Health status of Pomatoschistus microps populations in relation to pollution and natural stressors: implications for ecological risk assessment. Biomarkers, 17(1), 62-77. https://doi.org/10.3109/1354750X.2011.638442.

38. Habig, W. H., Pabst, M. J., \& Jakoby, W. B. (1974). Glutathione S-transferasesthe first enzymatic step in mercapturic acid formation. The Journal of Biological Chemistry, 249, 7130-7139.

39. Hagger, J. A., Jones, M. B., Leonard, D. R. P., Owen, R., \& Galloway, T. S. (2006). Biomarkers and integrated environmental risk assessment: are there more questions than answers? Integrated Environmental Assessment and Management, 2, 312-329. https://doi.org/10.1002/ieam.5630020403.

40. Halliwell, B., \& Gutteridge, J. M. C. (1999). Free radicals in biology and medicine. Oxford: Oxford University Press.

41. Hanna Instruments. (2014). C $99 \&$ \& 200 series multiparameter bench photometers. Instruction manual. http://www.hannacan.com/PDF/manC99.pdf. Accessed September 2014.

42. Harrington, J., \& Born, M. (2000). Measuring the health of California streams and rivers - a methods manual for water resource professionals, citizen monitors and natural resource students. California: Sustainable Land Stewardship International Institute.

43. Hollander, M., \& Wolfe, D. A. (1973). Non-parametric statistical methods. New York: John Wiley \& Sons.

44. INAG. (2008). Tipologia de rios em Portugal Continental no âmbito da implementação da Diretiva Quadro da Água, I- caracterização abiótica. Ministério do ambiente, do ordenamento do território e do desenvolvimento regional, 
Instituto da Água, I.P. https://www.apambiente.pt/dqa/assets/tipologia-de-riosem-portugal continental.pdf. Accessed September 2014 (in Portuguese).

45. INAG (2009). Critérios para a classificação do estado das massas de água superficiais - rios e albufeiras. Ministério do ambiente, do ordenamento do território e do desenvolvimento regional, Instituto da Água, I.P. https://www.apambiente.pt/dqa/assets/crit\%C3\%A9riosclassifica\%C3\%A7\%C3 \%A3o-rios-e-albufeiras.pdf. Accessed September 2014 (in Portuguese).

46. Jin, X., Zha, J., Xu, Y., Giesy, J. P., \& Wang, Z. (2012). Toxicity of pentachlorophenol to native aquatic species in the Yangtze River. Environmental Science and Pollution Research, 19, 609-618. https://doi.org/10.1007/s11356011-0594-1.

47. Jo, S. H., Son, M. K., Koh, H. J., Lee, S. M., Song, I. H., Kim, Y. O., Lee, Y. S., Jeong, K. S., Kim, W. B., Park, J. W., Song, B. J., \& Huhe, T. L. (2001). Control of mitochondrial redox balance and cellular defense against oxidative damage by mitochondrial NADP(+)-dependent isocitrate dehydrogenase. Journal of Biological Chemistry, 276, 16168-16176. https://doi.org/10.1074/jbc.M010120200.

48. Jones, C. (2008). Taxonomic sufficiency: the influence of taxonomic resolution on freshwater bioassessments using benthic macroinvertebrates. Environmental Reviews, 16(NA), 45-69. https://doi.org/10.1139/A07-010.

49. Kaya, H., Akbulut, M., Selvi, K., Ileri, B., \& Duysak, M. (2014). Heavy metal accumulation, biomarker responses and sensitivity to oxidative stress in Isopoda Asellus aquaticus from Saricay Creek (Canakkale-Turkey). Ekoloji, 23(91), 8-15. https://doi.org/10.5053/ekoloji.2014.912.

50. Kvičalová, M., Doubravová, P., Jobánek, R., Jokešová, M., Ocenášková, V., Süssenbeková, H., \& Svobodová, A. (2012). Application of different extraction methods for the determination of selected pesticide residues in sediments. Bulletin of Environmental Contamination and Toxicology, 89, 21-26. https://doi.org/10.1007/s00128-012-0622-y. 
51. Lam, P. K. S. (2009). Use of biomarkers in environmental monitoring. Ocean Coast Manage, 52, 348-354. https://doi.org/10.1016/j.ocecoaman.2009.04.010.

52. Le, S., Josse, J., \& Husson, F. (2008). FactoMineR: an R package for multivariate analysis. Journal of Statistic Software, 25(1), 1-18. https://doi.org/10.18637/jss.v025.i01.

53. Li, H., Wei, Y., Lydy, M. J., \& You, J. (2014). Inter-compartmental transport of organophosphate and pyrethroid pesticides in South China: implications for a regional risk assessment. Environmental Pollution, 190, 19-26. https://doi.org/10.1016/j.envpol.2014.03.013.

54. Liu, H. P., Hershler, R., \& Clift, K. (2003). Mitochondrial DNA sequences reveal extensive cryptic diversity within a western American springsnail. Molecular Ecology, 12, 2771-2782. https://doi.org/10.1046/j.1365-294X.2003.01949.x.

55. Lushchak, V. I. (2011). Environmentally induced oxidative stress in aquatic animals. Aquatic Toxicology, 101, 13-30. https://doi.org/10.1016/j.aquatox.2010.10.006.

56. Marshall, J. C., Steward, A. L., \& Harch, B. D. (2006). Taxonomic resolution and quantification of freshwater macroinvertebrate samples from an Australian dryland river: the benefits and costs of using species abundance data. Hydrobiologia, 572, 171-194. https://doi.org/10.1007/s10750-005-9007-0.

57. Martinez-Haro, M., Beiras, R., Bellas, J., Capela, R., Coelho, J. P., Lopes, I., Moreira-Santos, M., Reis-Henriques, A. M., Ribeiro, R., Santos, M. M., \& Marques, J. C. (2015). A review on the ecological quality status assessment in aquatic systems using community based indicators and ecotoxicological tools: what might be the added value of their combination? Ecological Indicators, 48, $8-16$.

58. Masiá, A., Campo, J., Vázquez-Roig, P., Blasco, C., \& Picó, Y. (2013). Screening of currently used pesticides in water, sediments and biota of the Guadalquivir River Basin (Spain). Journal of Hazardous Materials, 263(Part 1), 95-104. https://doi.org/10.1016/j.jhazmat.2013.09.035. 
59. Masiá, A., Campo, J., Navarro-Ortega, A., Barceló, D., \& Picó, Y. (2015). Pesticide monitoring in the basin of Llobregat River (Catalonia, Spain) and comparison with historical data. Science of the Total Environment, 503-504, 5868. https://doi.org/10.1016/j.scitotenv.2014.06.095.

60. Menetrey, N., Oertli, B., Sartori, M., Wagner, A., \& Lachavanne, J. B. (2008). Eutrophication: are mayflies (Ephemeroptera) good bioindicators for ponds? Hydrobiologia, 597, 125-135. https://doi.org/10.1007/s10750-007-9223-x.

61. Minutoli, R., Granata, A., \& Guglielmo, L. (2013). Potential use of ecotoxicological biomarkers in Serratella ignita (Ephemeroptera) larvae for Alcantara river (Sicily, Italy) water quality assessment. Journal of Limnology, 72, 394-399. https://doi.org/10.4081/jlimnol.2013.e32.

62. Montuori, P., Aurino, S., Nardone, A., Cirillo, T., \& Triassi, M. (2015). Spatial distribution and partitioning of organophosphates pesticide in water and sediment from Sarno River and Estuary, Southern Italy. Environmental Science and Pollution Research 22, 8629-8642. https://doi.org/10.1007/s11356-014-4016-z.

63. Montuori, P., Aurino, S., Garzonio, F., Sarnacchiaro, P., Polichetti, S., Nardone, A., \& Triassi, M. (2016). Estimates of Tiber River organophosphate pesticide loads to the Tyrrhenian Sea and ecological risk. Science of the Total Environment, 559, 218-231. https://doi.org/10.1016/j.scitotenv.2016.03.156.

64. Olsen, T., Ellerbeck, L., Fisher, T., Callaghan, A., \& Crane, M. (2001). Variability in acetylcholinesterase and glutathione S-transferase activities in Chironomus riparius Meigen deployed in situ at uncontaminated field sites. Environmental Toxicology and Chemistry, 20, 1725-1732. https://doi.org/10.1002/etc.5620200815.

65. Orbea, A., Ortiz-Zarragoitia, M., Sole, M., Porte, C., \& Cajaraville, M. P. (2002). Antioxidant enzymes and peroxisome proliferation in relation to contaminant body burdens of PAHs and PCBs in bivalve molluscs, crabs and fish from the Urdaibai and Plentzia estuaries (Bay of Biscay). Aquatic Toxicology, 58, 75-98. https://doi.org/10.1016/S0166-445X(01)00226-0. 
66. Payne, J. F., Mathieu, A., Melvin, W., \& Fancey, L. L. (1996). Acetylcholinesterase, an old biomarker with a new future? Field trials in association with two urban rivers and a paper mill in Newfoundland. Marine Pollution Bulletin, 32, 225-231. https://doi.org/10.1016/0025-326X(95)00112-Z.

67. Pestana, J. L. T., Loureiro, S., Baird, D. J., \& Soares, A. (2009). Fear and loathing in the benthos: responses of aquatic insect larvae to the pesticide imidacloprid in the presence of chemical signals of predation risk. Aquatic Toxicology, 93, 138149. https://doi.org/10.1016/j.aquatox.2009.04.008.

68. Pfrender, M. E., Hawkins, C. P., Bagley, M., Courtney, G. W., Creutzburg, B. R., Epler, J. H., Fend, S., Ferrington, L. C., Hartzwell, P. L., Jackson, S., Larsen, D. P., Levesque, C. A., Morse, J. C., Peterson, M. J., Ruiter, D., Schindel, D., \& Whiting, M. (2010). Assessing macroinvertebrate biodiversity in freshwater ecosystems: advances and challenges in DNA-based approaches. Quarterly Review of Biology, 85, 319-340. https://doi.org/10.1086/655118.

69. PGRH1. (2012). Plano de Gestão de Região Hidrográfica do Minho e Lima (RH1). Relatório de base. Ministério da Agricultura, Mar, Ambiente e Ordenamento de Território. https://www.apambiente.pt/. Accessed September 2014 (in Portuguese).

70. PGRH1. (2016). Plano de Gestão de Região Hidrográfica do Minho e Lima (RH1). Relatório de base. IP: Ministério da Agricultura, Mar, Ambiente e Ordenamento de Território. https://www.apambiente.pt/. Accessed September 2014 (in Portuguese).

71. PGRH3. (2016). Plano de Gestão de Região Hidrográfica do Douro (RH3). Relatório de base. IP: Ministério da Agricultura, Mar, Ambiente e Ordenamento de Território. https://www.apambiente.pt/. Accessed September 2014 (in Portuguese). 
72. Prat, N., Rieradevall, M., Barata, C., \& Munne, A. (2013). The combined use of metrics of biological quality and biomarkers to detect the effects of reclaimed water on macroinvertebrate assemblages in the lower part of a polluted Mediterranean river (Llobregat River, NE Spain). Ecological Indicators, 24, 167176. https://doi.org/10.1016/j.ecolind.2012.06.010.

73. Puertolas, L., Damasio, J., Barata, C., Soares, A., \& Prat, N. (2010). Evaluation of side-effects of glyphosate mediated control of giant reed (Arundo donax) on the structure and function of a nearby Mediterranean river ecosystem. Environmental Research, 110, 556-564. https://doi.org/10.1016/j.envres.2010.05.004.

74. R Core Team. (2017). R: A language and environment for statistical computing, reference index version. Vienna: $\mathrm{R}$ Foundation for Statistical Computing http://www.R-project.org/.

75. Ramos, A. S., Antunes, S. C., Gonçalves, F., \& Nunes, B. (2015). The Gooseneck Barnacle (Pollicipes pollicipes) as a candidate sentinel species for coastal contamination. Archives of Environmental Contamination and Toxicology, 66(3), 317-326. https://doi.org/10.1007/s00244-013-9978-1.

76. Raven, P. J., Fox, P., Everard, M., Holmes, N. T. H., \& Dawson, F. H. (1997). River Habitat Survey: A new system for classifying rivers according to their habitat quality. In P. J. Boon \& D. L. Howell (Eds.), Freshwater quality: Defining the indefinable? (pp. 215-234). Edinburgh: The Stationery Office.

77. Raven, P. J., Holmes, N. T. H., Charrier, P., Dawson, F. H., Naura, M., \& Boon, P. J. (2002). Towards a harmonized approach for hydromorphological assessment of rivers in Europe: a qualitative comparison of three survey methods. Aquatic Conservation Marine and Freshwater Ecosystems, 12, 405-424. https://doi.org/10.1002/aqc.536.

78. Raven, P. J., Holmes, N., Pádua, J., Ferreira, J., Hughes, S., Baker, L., Taylor, L., \& Seager, K. (2009). River Habitat Survey in Southern Portugal, results from 2009. Bristol: Environment Agency. 
79. Rikans, L. E., \& Hornbrook, K. R. (1997). Lipid peroxidation, antioxidant protection and aging. Biochimica et Biophysica Acta (BBA)-Molecular Basis of Disease, 1362, 116-127. https://doi.org/10.1016/S0925-4439(97)00067-7.

80. Rodrigues, A. P., Gravato, C., \& Guimarães, L. (2013). Involvement of the antioxidant system in differential sensitivity of Carcinus maenas to fenitrothion exposure. Environmental Science - Processes \& Impacts, 15, 1938-1948. https://doi.org/10.1039/c3em00367a.

81. Rodrigues, A. P., Santos, L. H., Ramalhosa, M. J., Delerue-Matos, C., \& Guimarães, L. (2015). Sertraline accumulation and effects in the estuarine decapod Carcinus maenas: importance of the history of exposure to chemical stress. Journal of Hazardous Materials, 283, 350-358. https://doi.org/10.1016/j.jhazmat.2014.08.035.

82. Rubal, M., Guilhermino, L. M., \& Medina, M. H. (2009). Individual, population and community level effects of subtle anthropogenic contamination in estuarine meiobenthos. Environmental Pollution, 157, 2751-2758. https://doi.org/10.1016/j.envpol.2009.04.026.

83. Saenz, L. A., Seibert, E. L., Zanette, J., Fiedler, H. D., Curtius, A. J., Ferreira, J. F., de Almeida, E. A., Marques, M. R. F., \& Bainy, A. C. D. (2010). Biochemical biomarkers and metals in Perna perna mussels from mariculture zones of Santa Catarina, Brazil. Ecotoxicology and Environmental Safety, 73, 796-804. https://doi.org/10.1016/j.ecoenv.2010.02.015.

84. Sahan, A., Kurutas, E. B., \& Altun, T. (2010). The determination of biochemical indicators (biomarkers) in the common carp (Cyprinus carpio) to the physicochemical parameters of the Ceyhan River (Adana-Turkey). Ekoloji, 19, 8-14. https://doi.org/10.5053/ekoloji.2010.762.

85. Santos, C. S. A., Monteiro, M. S., Soares, A., \& Loureiro, S. (2012). Characterization of cholinesterases in plasma of three Portuguese native bird species: application to biomonitoring. PLoS One, 7(3), e33975. https://doi.org/10.1371/journal.pone.0033975. 
86. Schulz, R., \& Liess, M. (2000). Toxicity of fenvalerate to caddisfly larvae: chronic effects of 1-vs 10-h pulse-exposure with constant doses. Chemosphere, 41, 15111517. https://doi.org/10.1016/S0045-6535(00)00107-7.

87. Siebel, A. M., Rico, E. P., Capiotti, K. M., Piato, A. L., Cusinato, C. T., Franco, T. M. A., Bogo, M. R., \& Bonan, C. D. (2010). In vitro effects of antiepileptic drugs on acetylcholinesterase and ectonucleotidase activities in zebrafish (Danio rerio) brain. Toxicology, 24, 1279-1284. https://doi.org/10.1016/j.tiv.2010.03.018.

88. Stohs, S. J., \& Bagghi, D. (1995). Oxidative mechanisms in the toxicity of metal ions. Free Radical Biology and Medicine, 18, 321-336. https://doi.org/10.1016/0891-5849(94)00159-H.

89. Tachet, H., Richoux, P., Bournaud, M., \& Usseglio-Polatera, P. (2002). Invertébrés d'eau douce. Paris: CNRS Edn (in French).

90. Ter Braak, C. J. F., \& Prentice, I. C. (1988). A theory of gradient analysis. Advances in Ecological Research, 18(C), 271-317. https://doi.org/10.1016/S0065-2504(08)60183-X.

91. Terrado, M., Lavigne, M. P., Tremblay, S., Duchesne, S., Villeneuve, J. P., Rousseau, A. N., Baceló, D., \& Tauler, R. (2009). Distribution and assessment of surface water contamination by application of chemometric and deterministic models. Journal of Hydrology, 369, 416-426. https://doi.org/10.1016/j.jhydrol.2009.02.030.

92. Vassault, A. (1983). Methods of enzymatic analysis. New York: Academic. $\underline{\text { Google Scholar }}$

93. Weiss, M., Macher, J. N., Seefeldt, M. A., \& Leese, F. (2014). Molecular evidence for further overlooked species within the Gammarus fossarum complex (Crustacea: Amphipoda). Hydrobiologia, 721, 165-184. https://doi.org/10.1007/s10750-013-1658-7.

94. Winston, G. W., \& Di Giulio, R. T. (1991). Prooxidant and antioxidant mechanisms in aquatic organisms. Aquatic Toxicology, 19, 137-161. https://doi.org/10.1016/0166-445X(91)90033-6. 


\section{Acknowledgements}

We are further grateful for the comments and suggestions of the editor and reviewer which helped to improve the manuscript.

\section{Funding}

The present study was supported by the Foundation for Science and Technology (FCT) through a PhD grant attributed to Rodrigues C. R. (SFRH/BD/87695/2012) and a postdoc fellowship attributed to Cruz V. F. (SFRH/BPD/109153/2015) and by the Strategic Funding UID/Multi/04423/2019 through national funds provided by the FCT and European Regional Development Fund (ERDF), in the framework of the programme PT2020. 